\title{
Modeling the ascent of sounding balloons: derivation of the vertical air motion
}

\author{
A. Gallice ${ }^{1}$, F. G. Wienhold ${ }^{1}$, C. R. Hoyle ${ }^{1, *}$, F. Immler ${ }^{2}$, and T. Peter ${ }^{1}$ \\ ${ }^{1}$ Institute for Atmospheric and Climate Science, Swiss Federal Institute of Technology, Zurich, Switzerland \\ ${ }^{2}$ Richard Assmann Observatory, German Meteorological Service (DWD), Lindenberg, Germany \\ *now at: Laboratory of Atmospheric Chemistry, Paul Scherrer Institut, Villigen, Switzerland
}

Received: 3 June 2011 - Published in Atmos. Meas. Tech. Discuss.: 23 June 2011

Revised: 21 September 2011 - Accepted: 6 October 2011 - Published: 20 October 2011

\begin{abstract}
A new model to describe the ascent of sounding balloons in the troposphere and lower stratosphere (up to $\sim 30-35 \mathrm{~km}$ altitude) is presented. Contrary to previous models, detailed account is taken of both the variation of the drag coefficient with altitude and the heat imbalance between the balloon and the atmosphere. To compensate for the lack of data on the drag coefficient of sounding balloons, a reference curve for the relationship between drag coefficient and Reynolds number is derived from a dataset of flights launched during the Lindenberg Upper Air Methods Intercomparisons (LUAMI) campaign. The transfer of heat from the surrounding air into the balloon is accounted for by solving the radial heat diffusion equation inside the balloon. In its present state, the model does not account for solar radiation, i.e. it is only able to describe the ascent of balloons during the night. It could however be adapted to also represent daytime soundings, with solar radiation modeled as a diffusive process. The potential applications of the model include the forecast of the trajectory of sounding balloons, which can be used to increase the accuracy of the match technique, and the derivation of the air vertical velocity. The latter is obtained by subtracting the ascent rate of the balloon in still air calculated by the model from the actual ascent rate. This technique is shown to provide an approximation for the vertical air motion with an uncertainty error of $0.5 \mathrm{~m} \mathrm{~s}^{-1}$ in the troposphere and $0.2 \mathrm{~m} \mathrm{~s}^{-1}$ in the stratosphere. An example of extraction of the air vertical velocity is provided
\end{abstract}

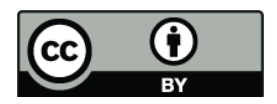

Correspondence to: A. Gallice (aurelien.gallice@gmail.com) in this paper. We show that the air vertical velocities derived from the balloon soundings in this paper are in general agreement with small-scale atmospheric velocity fluctuations related to gravity waves, mechanical turbulence, or other small-scale air motions measured during the SUCCESS campaign (Subsonic Aircraft: Contrail and Cloud Effects Special Study) in the orographically unperturbed mid-latitude middle troposphere.

\section{Introduction}

Sounding balloons are extensively used in meteorological forecasting and research, to the extent that several hundreds of them are sent daily into the atmosphere worldwide. They are mostly used to carry radiosondes aloft, enabling for the in situ recording of atmospheric variables with high temporal frequency and precision. This measurement technique stands among the most popular, for it is not subject to the same limitations as the majority of remote sensing instruments, such as decreasing accuracy with altitude or susceptibility to cloud cover.

Despite the wide usage of sounding balloons, rather limited effort has been put into the detailed modeling of their ascent. This results originally from the practice of storing radiosonde temperature, wind and humidity data only on a small number of so-called mandatory and significant levels (Alexander et al., 2010) with very coarse vertical resolution. Yet, for special cases radiosonde vertical ascent velocities have been analyzed in detail; e.g. Shutts et al. (1988) calculated the momentum flux of a single strong gravity wave

Published by Copernicus Publications on behalf of the European Geosciences Union. 
from fluctuations in balloon ascent velocities. However, Zink and Vincent (2001) state that smaller fluctuations can be due to measurement errors of radiosonde altitude or changing drag coefficient of the balloon, and recommend to calculate the vertical perturbation velocity from observed temperature fluctuations, assuming the intrinsic frequency of the contributing waves to derive the vertical momentum flux. Their statement nevertheless lacks support by evidences, and we expect their method to provide a low-accuracy estimation of the vertical air motion.

In an effort to obtain information also about atmospheric smaller scale wave activity the World Climate Research Program's (WCRP's) Stratospheric Processes and their Role in Climate (SPARC) project started to save the high-resolution radiosonde data (Hamilton and Vincent, 1995), archiving them at the SPARC Data Center. ${ }^{1}$ Still, a general modeling approach for radiosonde ascents in dependence on the state of the atmosphere is lacking.

A coarse modeling approach for sounding balloon ascents assuming constant ascent velocities has been used recently to improve the precision of the "match" technique (Engel, 2009). The latter consists in probing the same air parcel twice using two sounding balloons launched at different times (typically a few hours apart) and locations (typically tens to hundreds of kilometers apart) in order to obtain information on the time evolution of the air parcel's properties, e.g. with respect to gases, aerosols or cloud particles. The match technique has been used in the past to compute ozone loss rate in the lower stratosphere at the poles (Rex et al., 1999), but the ozone match flights did not rely on the use of a balloon ascent model; the procedure consisted in launching the first balloon, then precisely forecasting the trajectories of the air parcels measured by the ozone sonde, and finally launching a second balloon from a location downstream in order to measure the air parcel a second time. In order to improve the quality for the match by the second sounding, a new procedure involving balloon ascent modeling has been proposed recently (Engel, 2009). Assuming a constant ascent rate of $5 \mathrm{~m} \mathrm{~s}^{-1}$ for the balloon superimposed on weather forecast or analysis data, this technique is currently used to study the evolution of supersaturations of water vapor with respect to ice in cirrus clouds, which should eventually lead to a better understanding of the role of cirrus clouds in climate change.

As the interest in sounding balloon modeling has rejuvenated only recently, there are surprisingly few more precise model attempts. One is the model recently proposed by Wang et al. (2009) enabling the extraction of the air vertical velocity from radiosonde data. Their method is based on a decomposition of the balloon ascent rate into a contribution representing the balloon ascent in still air and a contribution representing vertical air motion. The balloon ascent rate in the absence of vertical winds is computed using a model and

\footnotetext{
${ }^{1}$ http://www.sparc.sunysb.edu/html/hres.html
}

the radiosonde data. Air vertical velocity is then obtained by subtracting the ascent rate in still air from the actual ascent rate. Wang et al. discuss the advantages of this method over other techniques aimed at deriving the air vertical velocity. Their model for the ascent of a sounding balloon in still air is based on the balloon's momentum conservation equation. From this equation, they obtain an expression of the balloon ascent rate in still air as a function of the balloon volume and of the drag coefficient. The balloon volume change with altitude is computed from the balloon volume at ground by assuming thermal equilibrium with ambient air at all times during the ascent. The values of the drag coefficient - taken as constant above $5 \mathrm{~km}$ altitude - and of the balloon volume at ground are optimized for each flight so as to minimize the median departure of the modeled ascent rate in still air from the actual ascent rate.

Other ascent models have been developed for different types of balloons, especially zero-pressure balloons (Musso et al., 2004; Palumbo, 2007). These models often involve a thorough treatment of the radiative and convective transport of heat inside the balloon. The most advanced ones take geometric factors and the variation of the balloon drag coefficient with altitude into account (Palumbo, 2007). These models can, however, not be applied to the case of sounding balloons, since they rely on empirical relations - relating for example the drag coefficient to the Reynolds and Froude numbers - which are valid for zero-pressure balloons only. As a matter of fact, the latter differ from the sounding balloons with respect to at least two important points: (a) their size and their payload weight are of the order of 30 to 70 times higher, hereby providing them a much stronger inertia and diminishing consequently their sensibility to atmospheric disturbances; and (b) their envelope is not close to spherical but rather of a much more complex shape, thereby significantly influencing the dynamics of their drag coefficient.

In the present work, a model for the ascent of a sounding balloon in still air is developed, going beyond the work by Wang et al. (2009) by taking into account both the variation of the balloon drag coefficient with altitude and the heat imbalance between the balloon and the ambient air. In order to keep the model manageable, three major assumptions are made. Firstly, the balloon is approximated by an almost spherical bubble of gas, the latter being assumed to follow the ideal gas law. This approximation subtends that the balloon envelope is not resolved in the model, which implies that the pressure inside and outside of the balloon are considered to be equal. It should be noted that the balloon shape is not restricted to a perfect sphere so as to account for the effect of the air flow around the balloon and the presence of the payload. Secondly, it is assumed that the process responsible for the propagation of heat inside the balloon can be described as diffusion. This comprises not only molecular diffusion, but also convection and radiative heat transfer, which are both assumed to be representable by diffusive 
laws. One consequence of this approximation is that only night flights can be modeled accurately. Thirdly, the temperature distribution inside the balloon is assumed to be spherically symmetric. The permissibility of this approximation is granted by the fact that deviations of the balloon shape from spherical remain limited. Despite these assumptions, the present model is expected to enable more precise balloon trajectory forecasts and characterizations of the air vertical velocity than other currently available models.

The theoretical background underpinning the balloon ascent model is developed in Sect. 2. In Sect. 3, the ascent model is described in detail. Its evaluation and a discussion of its application to the derivation of the air vertical velocity are presented in Sect. 4. Section 5 provides a conclusion and a discussion of potential improvements to the present model.

\section{Theoretical background}

\subsection{Balloon ascent rate}

The expression of the ascent rate of the balloon in still air is derived from the balance between the "free lift", $F_{\mathrm{FL}}$, and the drag force, $F_{\mathrm{D}}$ (Wang et al., 2009). The free lift corresponds to the net upward force acting on the balloon and is expressed as the difference between the buoyancy force and the total weight of the balloon (Yajima et al., 2009),

$F_{\mathrm{FL}}=\left(\rho_{\mathrm{a}} V-m_{\mathrm{tot}}\right) g$,

where $\rho_{\mathrm{a}}$ denotes the ambient air mass density, $V$ the balloon volume, $m_{\text {tot }}$ the balloon total mass - namely the sum of the respective masses of the balloon envelope, of the lifting gas and of the payload - and $g$ the acceleration due to gravity at the surface of the Earth. The expression for the drag force in still air reads

$F_{\mathrm{D}}=\frac{1}{2} c_{\mathrm{D}} \rho_{\mathrm{a}} S v_{z}^{2}$,

where $c_{\mathrm{D}}$ refers to the drag coefficient, $S$ to the reference area and $v_{z}$ to the balloon ascent rate in still air. The reference area can be chosen arbitrarily, so that $c_{\mathrm{D}}$ is a priori not uniquely defined for a given drag force. In this study, $S$ is chosen as the cross-sectional area of the sphere with same volume as the balloon. This choice follows the standard definition of the reference area for non-spherical objects (Loth, 2008). The advantage of this choice is that the departure of the balloon shape from spherical is entirely captured and described by the drag coefficient only. Denoting by $R$ the radius of the volume-equivalent sphere, $S$ and $V$ can be written as: $\pi R^{2}$ and $(4 / 3) \pi R^{3}$, respectively.

The expression of $v_{z}$ is obtained by equating Eqs. (1) and (2),

$v_{z}=\sqrt{\frac{8 R g}{3 c_{\mathrm{D}}}\left(1-\frac{3 m_{\mathrm{tot}}}{4 \pi \rho_{\mathrm{a}} R^{3}}\right)}$, where $V$ and $S$ have been replaced by their respective expressions as a function of the volume-equivalent sphere radius, $R$, hereafter called "balloon effective radius." Provided that $m_{\text {tot }}$ is known and that $\rho_{\mathrm{a}}$ can be determined using either a numerical weather forecast (in the case of Eq. (3) being used to forecast the balloon trajectory) or using the radiosonde data recorded during the balloon ascent (in the case of Eq. (3) being used a posteriori for the derivation of the vertical air motion), the computation of $v_{z}$ from Eq. (3) still requires the knowledge of $R$ and $c_{\mathrm{D}}$. The balloon effective radius, as a result of the decreasing ambient air pressure, increases during the balloon ascent. If the expansion of the balloon volume was treated as a purely adiabatic process, the temperature difference between the ambient air and the balloon would continue to increase with altitude, for the environmental lapse rate is smaller than the adiabatic lapse rate. As a consequence, heat transfer from the ambient air into the balloon must also be taken into account if the variation of the balloon volume with altitude is to be determined physically. Heat transfer is resolved in the present case by solving the radial heat diffusion equation inside the balloon with a prescribed Dirichlet boundary condition at the balloon surface, as discussed in more detail in Sect. 2.2. The dynamics of the drag coefficient are discussed in Sect. 2.3.

\subsection{Heat diffusion inside the balloon}

The variation of the balloon effective radius $(R)$ with altitude results from both adiabatic expansion and heat transfer from the surrounding air into the balloon. The heat flux at the balloon surface is assumed to propagate inside the balloon volume by means of diffusion (see Sect. 1). In our model applications we restrict heat diffusion to be only molecular; the case where also eddy diffusion or convection are assumed to take place is discussed in Sect. 5. The temperature distribution inside the balloon, $T_{\mathrm{b}}(r, t)$, is assumed to be spherically symmetric and therefore to obey the radial heat diffusion equation (Carslaw and Jaeger, 1959),

$\frac{\partial T_{\mathrm{b}}}{\partial t}=\frac{\langle D\rangle}{R^{2}} \frac{1}{r^{2}} \frac{\partial}{\partial r}\left(r^{2} \frac{\partial T_{\mathrm{b}}}{\partial r}\right)$,

where $\langle D\rangle=\left\langle\kappa /\left(\rho_{\mathrm{b}} c_{p}\right)\right\rangle$ is the mean molecular heat diffusion coefficient averaged over the balloon volume, $r \in[0,1]$ denotes the radial coordinate non-dimensionalized by the balloon effective radius $(R)$ and $t$ refers to time. The normalization of the radial coordinate by $R$ simplifies the discussion of the model in Sect. 3. In the expression for the mean molecular heat diffusion coefficient, $\kappa$ refers to the lifting gas thermal conductivity, which is a known function of $T_{\mathrm{b}}$ (see e.g. Vargaftik et al., 1994, for the thermal conductivity of hydrogen and helium), $\rho_{\mathrm{b}}$ denotes the lifting gas mass density, deduced from $T_{\mathrm{b}}$ and the pressure using the perfect gas law, $c_{p}$ is the lifting gas specific heat capacity at constant pressure, taken here as constant, and $\langle\cdot\rangle$ refers to the average over the balloon volume. Regarding the boundary 
conditions, the lifting gas temperature at the balloon surface is assumed to be the same as the ambient air temperature, viz. $T_{\mathrm{b}}(r=1)=T_{\mathrm{a}}$. At the balloon center, the heat flux is imposed to vanish as a result of the symmetry of the problem, viz. $\left(\partial T_{\mathrm{b}} / \partial r\right)_{r=0}=0$.

Equation (4) presents a simplification, because the work and convection terms associated with the expansion of the gas are not considered. This avoids the requirement of using the mass conservation equation to close the system. It should be noted that the suppression of the expansion terms is equivalent to considering the gas as incompressible; in particular, it implies that the balloon effective radius remains constant while heat diffuses. This constraint is justified for the small time intervals (0.3-1 s, see Sect. 3) over which heat diffusion is evaluated using Eq. (4). At the end of each time interval, both the temperature distribution and the balloon effective radius are corrected to account for the gas expansion. The correction procedure will be described later in Sect. 3.

The molecular heat diffusion coefficient is approximated by its average over the balloon volume. This approximation constitutes a correction to the fact that heat convection is not taken into account in the present model. In addition, $\langle D\rangle$ is assumed to be constant over time intervals of a few seconds. This is granted because so short time intervals correspond to just a few percent of the characteristic time of diffusion (see discussion below). The assumption of constant $\langle D\rangle$ is particularly valuable since it turns Eq. (4) into a simple partial differential equation.

Under these conditions Eq. (4) is amenable to an analytical solution (Carslaw and Jaeger, 1959). The latter is expressed as a Fourier series whose coefficients involve the computation of integrals over the radial coordinate $r$, requiring significant computational effort. In the balloon ascent model, we rather solve Eq. (4) numerically by the Finite Element Method. For a description of the Finite Element Method applied to the problem of heat diffusion, see e.g. Lewis et al. (1996). The analytical solution is however useful in two different aspects. Firstly, it can be used to estimate the magnitude of the characteristic time of diffusion, $\tau=R^{2} /\left(\pi^{2} D\right)$. The estimate is calculated in Appendix A. It is found that $\tau$ decreases from $\sim 900 \mathrm{~s}$ at ground to $\sim 300 \mathrm{~s}$ at $30 \mathrm{~km}$ altitude, validating that the temperature distribution inside the balloon varies little over time intervals of a few seconds. Secondly, the analytical solution can be used to study the convergence of the finite element solution in simple cases of reference. Evidences for the convergence of the numerical solution are provided in Appendix B.

\subsection{Balloon drag coefficient}

In this section, the dynamics of the drag coefficient of a perfect sphere are detailed first. These are then used as a basis for the discussion of the drag coefficient of spheroids, aimed at illustrating the case of almost spherical objects. From these two steps, the current knowledge on the drag coefficient of objects placed in a cross-flow is found to be insufficient to precisely model the balloon ascent. To compensate for this, information on the drag coefficient of sounding balloons is extracted from experimental flights in a third step.

\subsubsection{Drag coefficient of a perfect sphere}

As pointed out by numerous experimental studies (e.g., Son et al., 2010), the drag coefficient of a perfect sphere is mainly a function of two other dimensionless numbers, namely the Reynolds number, $R e$, and the free-stream turbulence intensity, $T u$ (see below). The Reynolds number is a measure of the ratio of inertial energy, $\rho_{\mathrm{a}} v_{z}^{2}$, to viscous energy, $\mu v_{z} / R$, where $\mu$ is the dynamic viscosity of the fluid. Consequently, $R e=\rho_{\mathrm{a}} R v_{z} / \mu$ quantifies the relative importance of these two types of energies for given flow conditions. In the case of a sounding balloon, whose typical effective radius is of the order of $1 \mathrm{~m}$ at ground and mean ascent rate of the order of $5 \mathrm{~m} \mathrm{~s}^{-1}$, the Reynolds number decreases from $\sim 8-9 \times 10^{5}$ at ground to $\sim 6-9 \times 10^{4}$ at $30 \mathrm{~km}$ altitude. In this range of Reynolds numbers, the drag coefficient of perfect spheres undergoes a sudden increase, referred to as the drag crisis, as the Reynolds number decreases and experiences a transition from the super- to the sub-critical regimes (Vennard and Street, 1976). The drag crisis is explained by a transition of the boundary layer from turbulent to laminar as $R e$ decreases, which advances the position of the boundary layer separation point upstream at the surface of the sphere (Vennard and Street, 1976). In summary, for a balloon ascending in the atmosphere the sequence of dynamical changes is as follows: height increases $\rightarrow$ air density decreases $\rightarrow R e$ decreases $\rightarrow$ boundary layer turns from turbulent to laminar $\rightarrow$ boundary layer detachment point advanced upstream at the surface of the balloon $\rightarrow$ drag coefficient increases. According to Achenbach (1972), the critical Reynolds number at which the drag crisis occurs, lies in the range 3.5$3.8 \times 10^{5}$ in the case of a negligible free-stream turbulence intensity $(T u=0.45 \%)$. His experimental curve obtained from a rigid sphere held fixed in space in a cross-flow wind tunnel is partly reproduced in Fig. 1. It can be observed that in the super-critical regime $\left(\operatorname{Re}>3.5 \times 10^{5}\right)$ the drag coefficient slightly decreases from its starting value of $\sim 0.1$ at $R e=10^{6}$, then rapidly increases during the drag crisis, before stabilizing in the sub-critical regime $\left(\operatorname{Re}<3.5 \times 10^{5}\right)$ where it remains almost constant at a value of $\sim 0.5$.

The free-stream turbulence intensity, $T u$, is defined as the ratio of the standard deviation of the incident air velocity fluctuations to the mean incident air velocity (e.g., Son et al., 2010). Contrary to $R e, T u$ is purely a property of the fluid. As the free-stream turbulence intensity is increased, the critical Reynolds number is observed to shift to lower values (Son et al., 2010). This is explained by the turbulence intensity delaying the boundary layer transition from turbulent to laminar, hereby leading to a drag crisis at lower Reynolds numbers. The experimental drag curves of Son et al. (2010) 


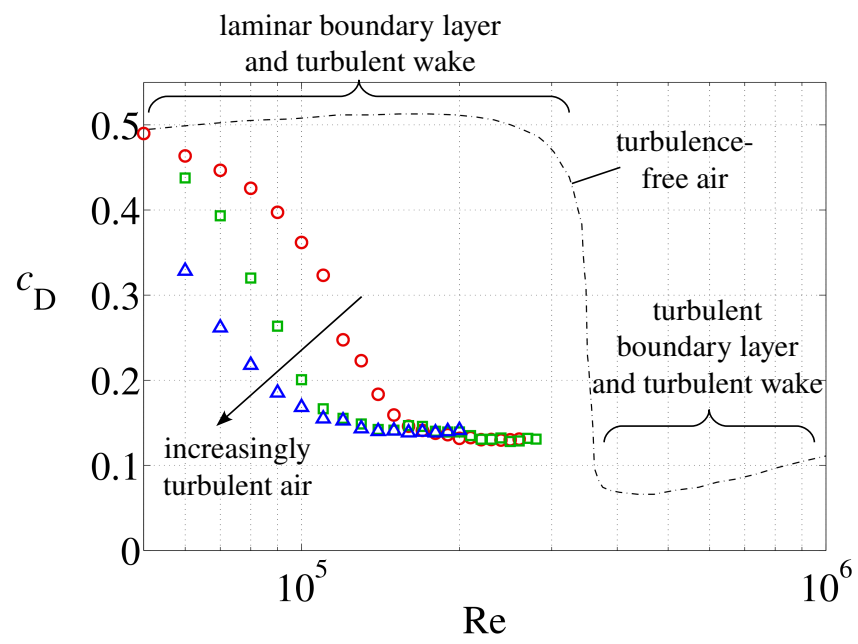

Fig. 1. Drag coefficient of a sphere as a function of the Reynolds number: $T u=0.45 \%(-\cdots-)$, data from Achenbach (1972); $T u=$ $4 \%(\bigcirc), T u=6 \%(\square), T u=8 \%(\Delta)$, data from Son et al. (2010).

characteristic for a sphere held fixed in space at three different $T u$ values are also reproduced in Fig. 1, where the term "drag curve" refers to the curve of $c_{\mathrm{D}}$ as a function of $R e$ at given $T u$. It can be observed that a level of free-stream turbulence as low as $4 \%$, which is a typical value of the turbulence intensity of the free troposphere (e.g., Hoyle et al., 2005), is sufficient to decrease the value of the critical Reynolds number by more than $50 \%$ as compared to the turbulence-free curve, leading to a decrease of $c_{\mathrm{D}}$ by as much as $70 \%$ in the range of Reynolds numbers $2-3 \times 10^{5}$. Likewise, the variation of $c_{\mathrm{D}}$ between the drag curves at $T u=4 \%$ and $T u=6 \%$ may reach more than $40 \%$ depending on the Reynolds number. It is concluded that the drag curve of a perfect sphere is extremely sensitive to the level of free-stream turbulence.

\subsubsection{Drag coefficient of a spheroid}

For a spheroid, the drag coefficient dependence on $R e$ qualitatively resembles that of a perfect sphere as a result of the similarity of both shapes (Loth, 2008). In particular, also the drag coefficient of a spheroid is a function of $R e$ and $T u$. It is expected to tend to the value for a perfect sphere as the respective lengths of the principal axes of the spheroid converge to the same value. Thus, the drag coefficient of a spheroid also depends on the departure of the spheroid shape from a perfect sphere. This departure is measured in terms of the aspect ratio, $E$, defined as the ratio of the length of the vertical symmetry axis to that of the horizontal axes of the spheroid. For example, Loth (2008) reports that the drag coefficient of an oblate spheroid with $E=0.5$ is about twice that of the volume-equivalent sphere for $2 \times 10^{3}<R e<3 \times 10^{5}$ and negligible $T u$.
To the best of the authors' knowledge, Eric Loth (2008) is the only author to report experimental investigations of the drag coefficient of spheroids at very high Reynolds numbers $\left(R e>10^{4}\right)$. He unfortunately considers only one single value for the aspect ratio, namely $E=0.5$. He also does not investigate the influence of the free-stream turbulence intensity on the drag curve. More importantly, his study does not extend beyond $R e>3 \times 10^{5}$, which leaves the entire supercritical regime unexplored to date. It should be noted that these last two limitations do not apply only to the work of Loth on the drag coefficient of spheroids, but also to all studies published to date on the drag coefficient of non-spherical objects. To compensate for this lack of knowledge, and since parameters other than $R e, T u$ and $E$ - such as unsteadiness or turbulence intensity length scale - are also known to affect the drag coefficient (e.g. Wang et al., 2009; Neve, 1986), an attempt is made here to derive a mean experimental drag curve for sounding balloons, based on a dataset of balloon flights. This attempt is expected to resolve also another principal complication, namely the fact that experimental investigations of drag coefficients normally let a heavy body fall freely in a viscous fluid or hold a solid body fixed in space and then expose it to a flow of the surrounding medium, e.g. in a wind tunnel. In such experiments detaching vortices in the wake of the particle affect very little the motion of the body, whose mass, due to the setup, appears to be extremely high. In contrast, a sounding balloon, whose mass is only a small fraction of that of the displaced air, is severely affected by the detaching vortices. As such, the analysis of a dataset of observed ascents appears to be the best way forward at the present time.

\subsubsection{Procedure for the derivation of a drag curve for sounding balloons from experimental flights}

The dataset is chosen from the flights which took place at Lindenberg (Germany) in 2008 during the Lindenberg Upper Air Methods Intercomparisons (LUAMI) flight campaign, whose main aim was to compare different airborne watervapor sounding methods (Immler, 2008). During the campaign, the masses of the payload (including the parachute) and the balloon envelope were measured before each flight, as well as the uplift mass; this allows for the balloon total mass, $m_{\mathrm{tot}}$, and the balloon radius at ground, $R(z=0)$, to be calculated. It should be mentioned that the uplift mass is defined as the value of the payload mass for which the free lift is equal to zero (see Sect. 2.1). Respective uncertainty errors of $\pm 100 \mathrm{~g}$ and $\pm 200 \mathrm{~g}$ in the measurements of the uplift and payload masses cannot be excluded, which in turn result in respective uncertainties of $\pm 200 \mathrm{~g}$ and $\pm 10^{-2} \mathrm{~m}$ in $m_{\text {tot }}$ and $R(z=0)$. During the flights, air temperature and pressure were measured every second by the radiosondes. The balloon altitude was also recorded at the same frequency by a GPS on board the radiosondes. Of the 27 balloons launched during the campaign, only the 15 released at night are kept in this analysis to enforce the assumption of negligible radiative 

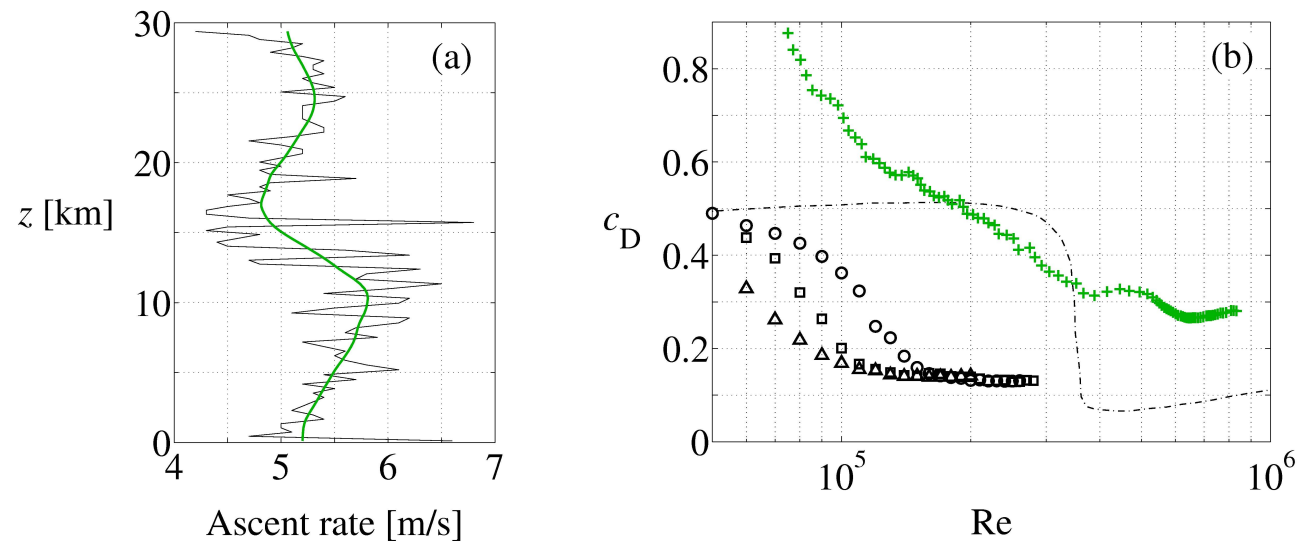

Fig. 2. Derivation of the experimental drag curve from LUAMI flight L007 launched on 7 November 2008 at 22:45 UTC. (a) The 60 s-low pass filtered ascent rate profile derived from the GPS data (-), and its mollified version using $\varepsilon=4 \mathrm{~km}(-)$; (b) experimental drag curve derived using the procedure described in Sect. $2.3(+)$. The curves of Achenbach (1972) and Son et al. (2010) for a perfect sphere are reported here for comparison (see Fig. 1).

heat transport into the balloon. A further selection is made removing five flights, three presenting strong evidence of defect (error in the reported value of the measured uplift mass or in the recording of the flight data) and two using a different type of sounding balloon. The dataset is therefore left with ten flights in total, all of which used the same type of sounding balloon, namely the TX1200 balloon from the Japanese company Totex. ${ }^{2}$

In order to derive a drag curve for sounding balloons from each of the ten selected experimental flights, the drag coefficient is calculated from Eq. (3) every minute of each flight as a function of $v_{z}, R$ and $\rho_{\mathrm{a}}$. To this end, the balloon radius is computed using the algorithm presented in Sect. 3, and the air mass density is determined from the 60-s low pass filtered atmospheric temperature and pressure data recorded during the balloon ascent. The challenge lies in the estimation of $v_{z}$, as only the ascent rate with respect to the ground, $v_{z, \mathrm{~g}}$, can be deduced from the radiosonde GPS data. The ascent rate in still air corresponds to the vertical velocity measured with respect to ambient air, which cannot be directly retrieved from the measurements. Thus, only an estimate of $v_{z}$ can be obtained by smoothing the profile of $v_{z, \mathrm{~g}}$ as a function of altitude. This procedure is based on the assumption of vertical air motion having a normal distribution with nearzero mean value (Wang et al., 2009). The smoothing process is performed by convoluting the vertical profile of $v_{z, \mathrm{~g}}$ with the mollifier $\eta_{\varepsilon}(z)$, where

$\eta_{\varepsilon}(z)= \begin{cases}(c / \varepsilon) \exp \left[\varepsilon^{2} /\left(z^{2}-\varepsilon^{2}\right)\right] & \text { if } z \in[-\varepsilon, \varepsilon], \\ 0 & \text { otherwise, }\end{cases}$

and the constant $c$ is chosen to ensure the unity of the integral of $\eta_{\varepsilon}$ (Salsa, 2008). The parameter $\varepsilon$ controls the spatial scale on which the profile of $v_{z, \mathrm{~g}}$ is smoothed. A value of $\varepsilon=4 \mathrm{~km}$

\footnotetext{
${ }^{2}$ http://www.totex.jp
}

is chosen here so as to ensure that gravity waves, whose typical vertical wavelengths are $2-5 \mathrm{~km}$ in the lower stratosphere (Fritts and Alexander, 2003), are properly removed from the measured ascent rate by the smoothing process. Other values $(\varepsilon=2 \mathrm{~km}$ and $\varepsilon=5 \mathrm{~km})$ have been investigated, but with negligible influence on the derived experimental drag curve (not shown).

An example of balloon ascent rate profile and of its associated mollified version is shown in Fig. 2a. The profile is observed to present an overall S-shape, which is typical for sounding balloons and can be simply explained by Eq. (3). Due to the diffusion of heat inside the balloon, the difference between the mean balloon temperature and the atmospheric temperature remains approximately constant over the troposphere and the stratosphere separately (not shown). Under this condition, it can be shown that the expression of $v_{z}$ in Eq. (3) is proportional to the $-1 / 6$ power of the atmospheric density (Yajima et al., 2009). This accounts for the fact that the balloon ascent rate increases with altitude over the troposphere and the stratosphere separately. The decrease in the ascent rate at the tropopause results from the sudden increase in the potential temperature. This can be interpreted as the balloon being suddenly colder than its environment and therefore decelerating, until its temperature difference with the surrounding atmosphere stabilizes and its ascent rate increases again as the $-1 / 6$ power of the atmospheric density. The decrease of the ascent rate above $25 \mathrm{~km}$ altitude observed in Fig. 2a is thought to result from another process. Shortly before bursting, the envelope of the balloon presents bubbles and excrescences on its surface due to an inhomogeneous distribution of the envelope material. This is expected to substantially increase the drag coefficient and consequently be at the origin of the balloon deceleration.

The drag curve corresponding to Fig. 2a and obtained by the aforementioned procedure is depicted in Fig. 2b. As 
expected from the aspherical shape of the balloon, this curve is observed to deviate significantly from those by Achenbach (1972) and Son et al. (2010) for a perfect sphere. However, the balloon drag curve presents a qualitative shape similar to the curves by Son et al. at $T u=6 \%$ and $T u=8 \%$. This suggests that the turbulence intensity of the atmosphere is of the order of $6 \%$ to $8 \%$, which is in the range of typical values for the free troposphere reported by Hoyle et al. (2005). Comparison of the balloon drag curve with the curves by Son et al. reveals that the drag coefficient of the balloon is on average three times higher than the one of its volume-equivalent sphere. This difference cannot be solely explained by the asphericity of the balloon. Indeed, Loth (2008) reports an increase of only $100 \%$ in the drag coefficient of a spheroid with $E=0.5$ as compared to a perfect sphere in the range of Reynolds number $0.5-3 \times 10^{5}$ and at negligible $T u$. The magnitude of this increase is expected to remain of roughly the same order at $T u>0$, while reducing with higher values of $E$. Therefore, the increase in $c_{\mathrm{D}}$ due to the limited departure of the balloon shape from spherical is clearly less than a factor of 2. This leaves part of the observed discrepancy between the balloon's and the perfect sphere's drag curves unexplained. Mainly three mechanisms are thought to be responsible: the pendulum effect of both the parachute and the payload attached to the balloon (Wang et al., 2009), the deformation of the balloon shape through the propagation of waves on its elastic envelope and the generation of vorticity in the wake of the balloon.

Regarding the latter mechanism, Govardhan and Williamson (2005) report the observation of two vortex threads detaching periodically from behind spheres placed in a cross-flow. In their experiments, the spheres are attached with a single tether to the upper wall of the wind tunnel so as to let them free to move in the horizontal plane (in both the directions parallel and perpendicular to the flow). The authors elegantly demonstrate that the periodically detaching vortex threads exert an oscillating force on the spheres in a direction transverse to the flow. Yet, Veldhuis et al. (2009) demonstrate that this force is usually not restricted to the plane transverse to the flow in the case of buoyant spheres rising freely in a Newtonian fluid. As a consequence, the component of this force in the direction of the spheres' motion is non-zero, which results in a so-called lift-induced drag. The latter adds to the drag predicted from the curves by Achenbach (1972) or Son et al. (2010) for a sphere held fixed in space. Thus, Veldhuis et al. estimate the apparent $c_{\mathrm{D}}$ of spheres rising freely to be higher by a factor 1.5 to 2 than expected from the standard drag curves alone. Unfortunately, the range of Reynolds number they consider is limited to the interval $1-2 \times 10^{3}$. However, we expect the generation of a lift-induced drag to be significant also for higher values of $R e$, and even more so for buoyant objects with non-spherical shape. This may account for a significant fraction of the unexpected drag depicted in Fig. 2 b.
From a physical point of view, the balloon drag curve pictured in Fig. $2 \mathrm{~b}$ is supported by the specifications of the balloon manufacturing company, according to which the balloon drag coefficient at $R e \sim 5-8 \times 10^{5}$ is in the range 0.2-0.3. Furthermore, this curve is in good agreement with the observations of Mapleson (1954), who reports an increase of up to $400 \%$ in the drag coefficient of sounding balloons as compared to a perfect sphere for $1.3 \times 10^{5}<R e<7 \times 10^{5}$.

\subsubsection{Reference drag curve for sounding balloons}

The drag curves derived from the ten LUAMI flights all present the same qualitative behavior as the curve described above. However, there are systematic offsets in $c_{\mathrm{D}}$ amongst these ten drag curves in the range $\pm 25 \%$, corresponding to \pm 0.15 absolute units in $c_{\mathrm{D}}$, as shown by the light gray curves in Fig. 3. We must attribute part of these offsets to errors in the estimated uplift and payload masses, i.e. in the preparatory measurements before each balloon launch during the LUAMI campaign. Indeed, an error of $100 \mathrm{~g}$ in the uplift mass shifts the corresponding drag curve by $6 \%$ through its effect on the values of $R(z=0)$ and $m_{\text {tot }}$ (not shown). Similarly, an error of $200 \mathrm{~g}$ in the payload mass would result in a shift of $7 \%$ in the balloon drag curves. Therefore, such errors might explain about half of the observed offsets in $c_{\mathrm{D}}$. The other half might be due to differences in the manufacturing process of the individual balloons, as invoked by Mapleson (1954) to explain the divergence of his results. While we cannot correct for these unknown differences in the manufacturing process, the confidence ranges of the uplift and payload masses can be taken into account in order to reduce the spread of the drag curves. To this end, $R(z=0)$ and $m_{\text {tot }}$ are adjusted within their accepted confidence ranges, minimizing the mean-square difference between the drag curves. The ten drag curves with adjusted offsets are pictured in green in Fig. 3. They are then fitted by a second-order polynomial in order to retrieve the single reference drag curve (blue line), which will be used in Sect. 3 to derive the balloon ascent rate in still air:

$c_{\mathrm{D}}=4.808 \times 10^{-2}(\ln R e)^{2}-1.406 \ln R e+10.490$.

The mean standard deviation of the ten experimental curves with respect to the polynomial fit is equal to $4.1 \times 10^{-2}$. Therefore, the values of the drag coefficient derived from the reference curve must be considered to have an uncertainty error of approximately \pm 0.04 .

Several important aspects of Eq. (6) should be stressed. First, the expression of the drag coefficient is observed not to depend on the turbulence intensity of the atmosphere. This results directly from the impossibility to determine $T u$ to the necessary precision from balloon flights, and implies that Eq. (6) accounts only for the mean profile of the atmospheric turbulence intensity. Deviations from this mean profile, such as the generation of turbulence intensity through gravity wave breaking, cannot be taken into account by the 


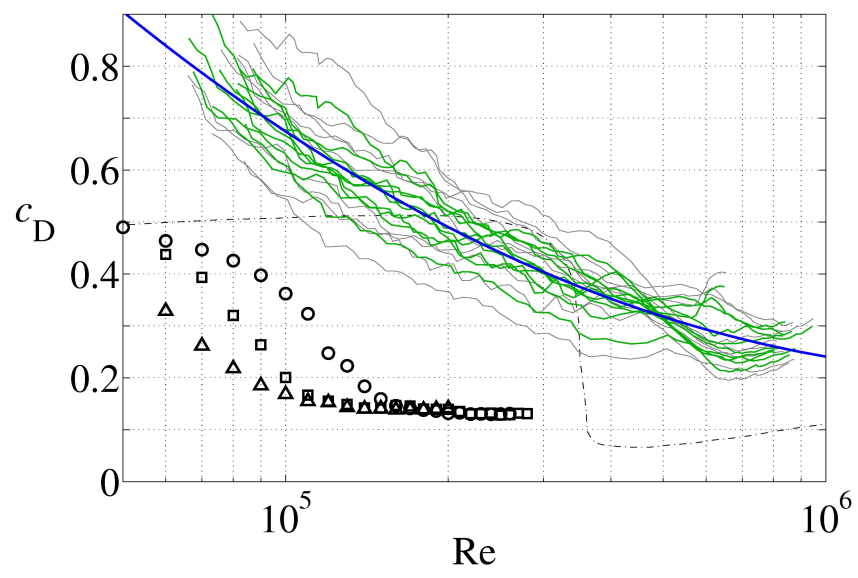

Fig. 3. Derivation of a reference drag curve for sounding balloons. (-) Experimental drag curves derived from the ten LUAMI balloon flights; (-) same but with adjusted values for $R(z=0)$ and $m_{\text {tot }}$; (-) fit to the ten experimental drag curves using a secondorder polynomial (Eq. 6). The curves of Achenbach (1972) (dashed) and Son et al. (2010) (symbols) for a perfect sphere are shown for comparison (see Fig. 1).

model. Second, $R e$ in Eq. (6) is a function of the balloon ascent rate (see Sect. 2.3.1). As a consequence, fluctuations in the balloon vertical velocity are explicitly taken into account in our drag calculation. Finally, it should be emphasized that Eq. (6) is valid only for TX1200 balloons launched at night. However, the procedure described above could be applied to any set of soundings featuring the required data. We have for example derived a reference drag curve for the two TX2000 balloons launched at night during the LUAMI campaign, and which were removed from the original dataset of night flights in Sect. 2.3.3. As compared to the TX1200 balloons, the values of the drag coefficient have been observed to be lower in the troposphere and much higher in the stratosphere (not shown), hereby pointing to the significant impact of the balloon shape on the drag curve.

\section{Balloon ascent model}

The balloon ascent model developed in this work aims to determine the ascent rate of sounding balloons in still air as a function of time. The model's time step is denoted by $\Delta t$ in the following and the corresponding increase in the balloon altitude by $\Delta z$; the two are related through the relation $\Delta z=v_{z} \Delta t+O\left(\Delta t^{2}\right)$.

A single step of the model comprises two parts:

1. the computation of the balloon effective radius and radial temperature distribution at time $t+\Delta t$ knowing their values at time $t$; and
2. the simultaneous determination of the drag coefficient and the balloon ascent rate in still air at time $t+\Delta t$ from Eq. (3).

For convenience of the reader, the computations performed in these two parts - to be detailed below - are summarized under the form of a pseudo-code in Fig. 4.

In order to increase the accuracy of the balloon's effective radius computation, part 1 uses substeps to resolve the balloon effective radius at intermediate times between $t$ and $t+\Delta t$. The intermediate times are computed using a subtime step, $\delta t$, chosen as a fixed fraction of the characteristic time of diffusion. This ensures that Eq. (4) is solved using a constant normalized time step, $\delta t / \tau$, during the whole balloon ascent. In the following discussion, let $\left\{t_{n}\right\}_{n=1, \ldots, N}$ be the set of intermediate times between $t$ and $t+\Delta t$, where $t_{n}=t+n \delta t$ and $N$ is the number of intermediate steps. In a single substep of part 1, the balloon effective radius at time $t_{n+1}$ is computed from the balloon effective radius at time $t_{n}$ in three stages (see left panel of Fig. 4):

(i) Adiabatic expansion of the balloon (pictured in Fig. 5a). In this stage, the balloon is considered to ascend from altitude $z\left(t_{n}\right)$ to altitude $z\left(t_{n+1}\right)$. Let $R^{*}$ and $T_{\mathrm{b}}{ }^{*}$ denote respectively the balloon effective radius and temperature distribution inside the balloon after the adiabatic expansion has taken place. Assuming that the pressure remains uniform inside the balloon and equilibrates with the ambient atmospheric pressure during the process,

$R^{*}=\left(\frac{p_{\mathrm{a}}\left(t_{n}\right)}{p_{\mathrm{a}}\left(t_{n+1}\right)}\right)^{1 / 3 \gamma} R\left(t_{n}\right)$,

$T_{\mathrm{b}}{ }^{*}(r)=\left(\frac{p_{\mathrm{a}}\left(t_{n}\right)}{p_{\mathrm{a}}\left(t_{n+1}\right)}\right)^{(1-\gamma) / \gamma} T_{\mathrm{b}}\left(r, t_{n}\right)$,

where $\gamma=c_{V} / c_{p}>1$ is the adiabatic index of the lifting gas $\left(c_{V}\right.$ is the lifting gas specific heat at constant volume) and Eq. (8) is valid for all $r \in[0,1]$. In the right-hand side of Eq. (8), $r$ denotes the radial coordinate normalized by $R\left(t_{n}\right)$, whereas in the left-hand side it is normalized by $R^{*}$.

(ii) Heat diffusion inside the balloon at constant pressure (pictured in Fig. 5b). As stated above in Sect. 2.2, this stage assumes the lifting gas to be incompressible; as a consequence, the balloon volume remains constant during the diffusion of heat. The mean heat diffusion coefficient is computed from the temperature distribution $T_{\mathrm{b}}{ }^{*}$ obtained in stage (i). Assuming that $\langle D\rangle$ remains constant, Eq. (4) is then solved numerically by the Finite Element Method using a time step of $\delta t=t_{n+1}-t_{n} . T_{\mathrm{b}}{ }^{*}$ is chosen as the initial temperature distribution, and the temperature at the balloon surface 


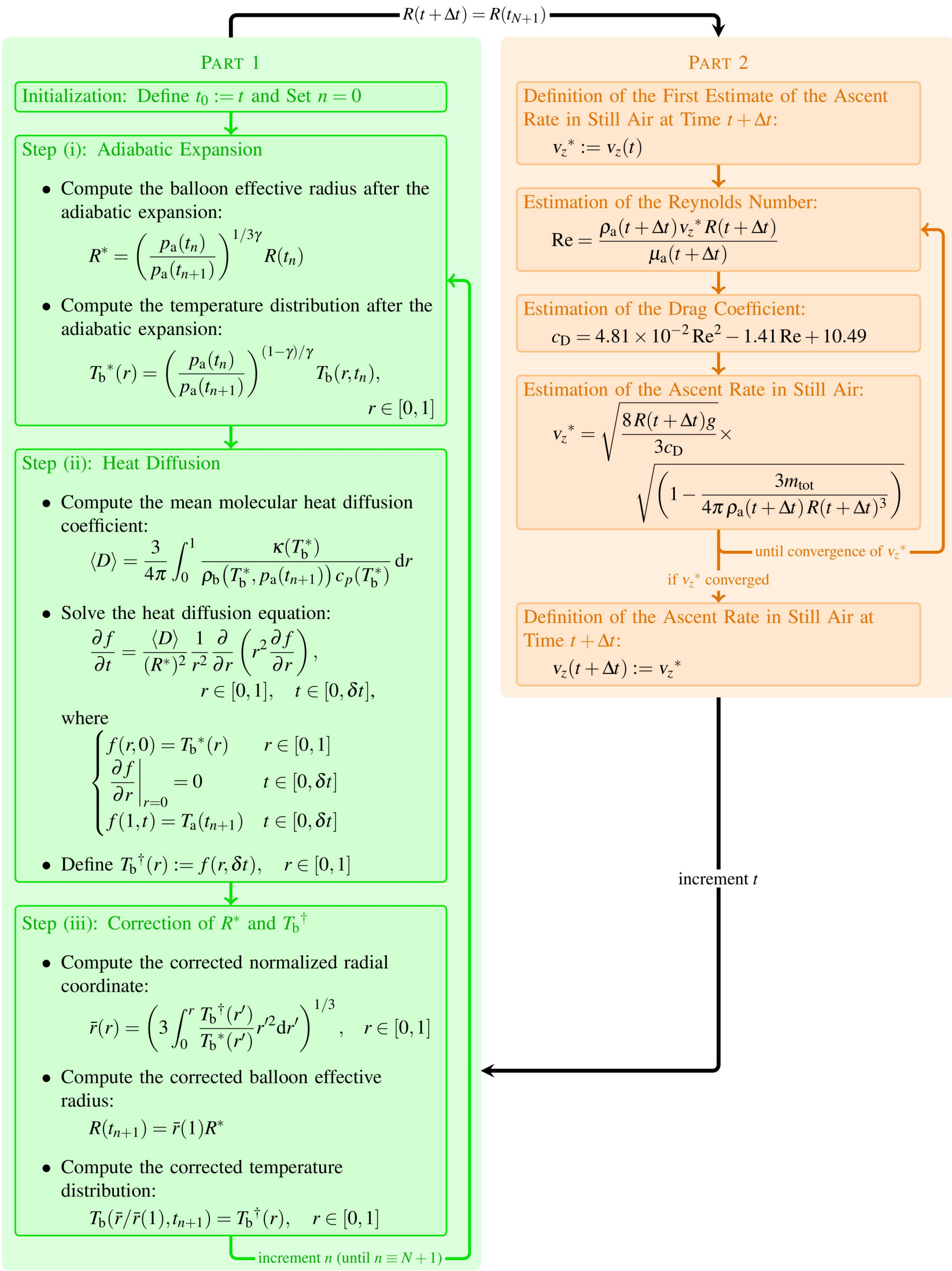

Fig. 4. Schematic representation of the different steps of the model. The notation is introduced in Sect. 3. 
(a)
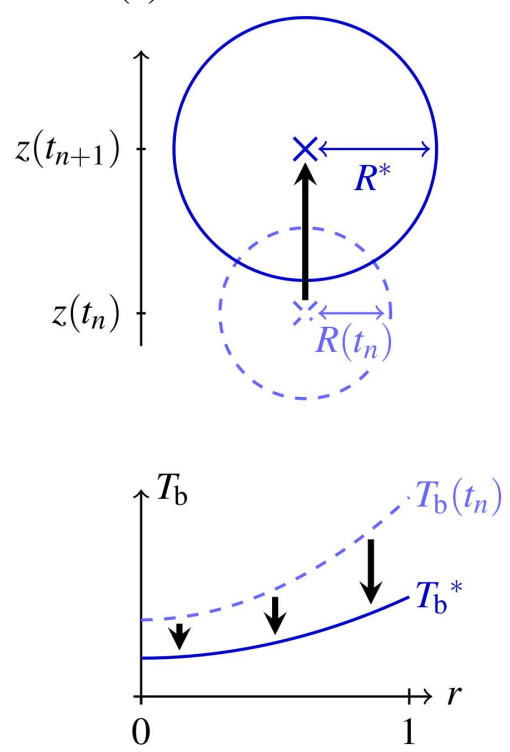

(b)

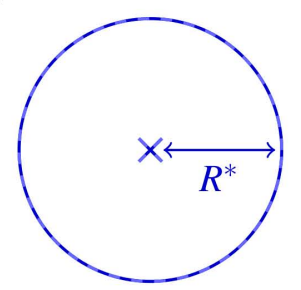

(c)
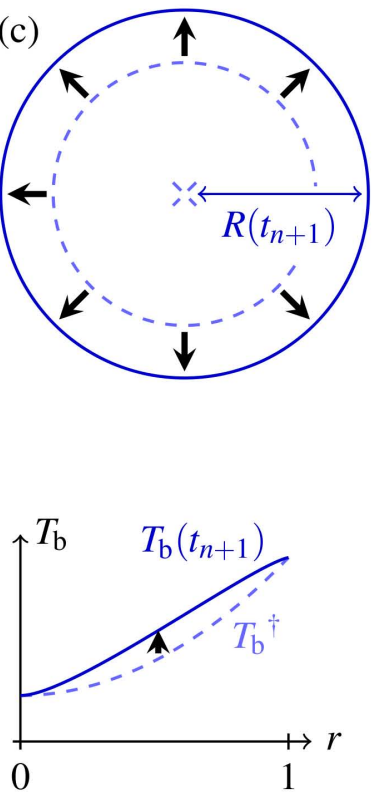

Fig. 5. Schematic representation of the three stages used in part 1 of the model to compute the balloon effective radius at time $t_{n+1}$ from the balloon effective radius at time $t_{n}$. The upper panel shows the evolution of the balloon altitude and effective radius at each step, the lower panel indicates the corresponding changes in the temperature distribution inside the balloon. The notation used in the figure is introduced in Sect. 3. (a) Adiabatic expansion of the balloon from altitude $z\left(t_{n}\right)$ to altitude $z\left(t_{n+1}\right)$. (b) Heat diffusion inside the balloon at constant pressure. (c) Correction to the balloon effective radius and temperature distribution.

is kept constant and equal to $T_{\mathrm{a}}\left(t_{n+1}\right)$. The temperature distribution at the end of the diffusion process is denoted by $T_{\mathrm{b}}^{\dagger}$.

(iii) Correction to the temperature distribution and balloon effective radius (pictured in Fig. 5c). To compensate for the above assumption of gas incompressibility during the diffusion of heat, $T_{\mathrm{b}}^{\dagger}$ and $R^{*}$ are corrected in this stage. To this end, let $\mathcal{S}$ be a spherical shell concentric to the balloon and whose normalized radius and infinitesimal thickness are denoted by $r(r<1)$ and $\mathrm{d} r$, respectively. The temperature of $\mathcal{S}$ is known from step (ii) to be $T_{\mathrm{b}}^{\dagger}(r)$. Given this configuration, the aim is to find the normalized radius and thickness, respectively denoted by $\bar{r}$ and $\mathrm{d} \bar{r}$, that $\mathcal{S}$ would have had if it had been let expand in step (ii). In such a case, its temperature would still have increased from $T_{\mathrm{b}}{ }^{*}(r)$ to $T_{\mathrm{b}}{ }^{\dagger}(r)$ as a result of heat diffusion. On the other hand, its pressure would have remained constant and equal to $p_{a}\left(t_{n+1}\right)$, while its volume would have increased from $4 \pi r^{2} \mathrm{~d} r$ to $4 \pi \bar{r}^{2} \mathrm{~d} \bar{r}$. Using the ideal gas law in association with the conservation of gas moles inside $\mathcal{S}$,

$\frac{4 \pi r^{2} \mathrm{~d} r}{T_{\mathrm{b}}^{*}(r)}=\frac{4 \pi \bar{r}^{2} \mathrm{~d} \bar{r}}{T_{\mathrm{b}}^{\dagger}(r)}$

In this equation, $\bar{r}$ is understood as a function of the uncorrected normalized radius $r$. Integrating Eq. (9) with respect to $r$,

$$
\bar{r}(r)=\left(3 \int_{0}^{r} \frac{T_{\mathrm{b}}^{\dagger}\left(r^{\prime}\right)}{T_{\mathrm{b}}{ }^{*}\left(r^{\prime}\right)} r^{\prime 2} \mathrm{~d} r^{\prime}\right)^{1 / 3} .
$$

It must be emphasized that both $\bar{r}$ and $r$ are normalized by the balloon effective radius $R^{*}$ resulting from step (i). Thus, the corrected balloon effective radius at time $t_{n+1}$ is given by

$R\left(t_{n+1}\right)=\bar{r}(1) R^{*}$,

and the corrected balloon temperature distribution at time $t_{n+1}$ reads

$T_{\mathrm{b}}\left(\bar{r} / \bar{r}(1), t_{n+1}\right)=T_{\mathrm{b}}^{\dagger}(r)$,

where $\bar{r}(1)$ is evaluated from Eq. (10).

Stages (i)-(iii) are repeated $N+1$ times until the balloon effective radius at time $t+\Delta t$ is evaluated. This terminates part 1 of the model.

In part 2, Eq. (3) is used to compute the balloon ascent rate in still air at time $t+\Delta t$ (see right panel of Fig. 4). The required air mass density is determined from the ambient atmospheric temperature and pressure, and the result obtained in part 1 is used for the balloon effective radius. The drag coefficient is determined from the reference second-order polynomial drag curve shown in Fig. 3. To this end, an estimation 
of the Reynolds number at time $t+\Delta t$ is derived from the balloon ascent rate at time $t$. The estimated $R e$ is then reported in the drag curve to estimate the drag coefficient. By inserting the latter in Eq. (3), a first estimate of $v_{z}(t+\Delta t)$ is obtained, which is subsequently used to refine the initial estimate of $R e$. This generates a loop, which is iterated until the convergence criterion is satisfied, namely until the relative variation of the ascent rate between two successive loops is less than $5 \times 10^{-4} \%$. At the end of part 2 of the model, the values of both $c_{\mathrm{D}}$ and $v_{z}$ at time $t+\Delta t$ are known.

The vertical profile of the balloon ascent rate in still air is derived by going through parts 1 and 2 of the model at each time step. The value of $\Delta t$ is fixed here to $1 \mathrm{~min}$, which corresponds to a vertical resolution of $\sim 300 \mathrm{~m}$. Based on a trade-off between computational time and the convergence study presented in Appendix B, the choice $\delta t=10^{-3} \tau$ is made, $\tau$ being computed at each step of the model. This results in a number of substeps $(N)$ increasing from $\sim 60$ to $\sim 180$ between ground level and $30 \mathrm{~km}$ altitude.

To reflect the uncertainty in the reference drag curve (see end of Sect. 2.3), three different runs of the model are recommended. The first run, corresponding to the reference case, uses the reference drag curve itself to calculate the most probable profile of the balloon ascent rate in still air. The two additional runs are aimed at determining the range of uncertainty in this profile. To this end, they are based on instances of the reference drag curve shifted along the $c_{\mathrm{D}}$-axis by $-\sigma_{c_{\mathrm{D}}}$ and $+\sigma_{c_{\mathrm{D}}}$, respectively, where $\sigma_{c_{\mathrm{D}}}=0.04$ denotes the uncertainty in the values of the drag coefficient derived from the reference drag curve (see Sect. 2.3).

In case the model is run after the balloon flight, advantage can be taken of the data collected during the ascent to improve the model in two respects. Firstly, the ascent rate derived from the GPS data can be used to correct the reference drag curve. The procedure consists in shifting the latter along the $c_{\mathrm{D}}$-axis so as to minimize the mean-square difference between the measured and modeled ascent rate profiles. This process is based on the assumption that the vertical wind follows a normal distribution with near-zero mean value, as supposed by Wang et al. (2009). Secondly, the uncertainty in the values of the drag coefficient derived from the shifted reference drag curve can be narrowed down. This uncertainty has been estimated for the general case in Sect. 2.3, where it has been defined as the mean standard deviation, $\sigma_{c_{\mathrm{D}}}$, of the difference between the experimental drag curves and the reference drag curve. In case the model is run after the actual flight, the experimental drag curve associated with the flight can be computed following the procedure described in Sect. 2.3. Only this experimental curve - instead of the ten of Fig. 3 - is then used to estimate the uncertainty in the values of $c_{\mathrm{D}}$ derived from the shifted reference drag curve. This uncertainty, denoted by $\sigma_{c_{\mathrm{D}}}^{*}$, corresponds to the standard deviation of the difference between the experimental drag curve associated to the flight and the shifted reference drag curve. It is observed that $\sigma_{c_{\mathrm{D}}}^{*}$ is generally lower as compared to $\sigma_{c_{\mathrm{D}}}$.

\section{Model evaluation and potential application}

\subsection{Model evaluation}

Due to the lack of available flight data with precisely measured uplift and payload masses, the validating set considered in this section is composed of the same ten LUAMI night flights used in Sect. 2.3 to derive the reference drag curve. Following the procedure described in the previous section, the latter is corrected for each flight so as to minimize the departure of the modeled ascent rate from the measured one. It should be noted that this section does not consider the payload and uplift masses measured before each flight during the LUAMI campaign, but rather the adapted values of these masses calculated in Sect. 2.3 to reduce the spread in the experimental curves.

An example of adapted drag curve is pictured in Fig. 6a; the corresponding profile of the balloon ascent rate in still air is shown in Fig. 6b. In this case, the correction of the reference drag curve allows for the decrease of the discrepancy between the modeled and measured ascent rates by $\sim 0.4 \mathrm{~m} \mathrm{~s}^{-1}$ below $10 \mathrm{~km}$ altitude. On the other hand, the balloon ascent rate in still air derived from the corrected reference drag curve appears to be overestimated in some regions, mostly in the lower troposphere below $2 \mathrm{~km}$ altitude and just below the tropopause between 10 and $12 \mathrm{~km}$ altitude. In these two altitude intervals, the Reynolds number is $7.5-8.5 \times 10^{5}$ and $4-5 \times 10^{5}$, respectively. As such, the apparent over-estimations of the ascent rate are related to the local maxima of the experimental drag curve at $R e=8.5 \times 10^{5}$ and $R e=4 \times 10^{5}$, respectively, which are unaccounted for by the (corrected) reference drag curve (see Fig. 6a). The latter considers lower drag coefficient values than the experimental drag curve at these Reynolds numbers, hereby leading to a lower drag force and consequently to a larger ascent rate in still air than expected from the smoothed observations. It must be emphasized that these apparent over-estimations of the ascent rate in still air may actually result from a local downward air motion affecting both the measured ascent rate and the experimental drag curve. Such a downdraft of the air would indeed slow down the actual ascent of the balloon and consequently increase its apparent drag coefficient, which could explain the observed difference between the reference and experimental drag curves. This could particularly be the case between $10 \mathrm{~km}$ and $12 \mathrm{~km}$ altitude, where the measured ascent rate is observed to drop below the lower uncertainty limit of the modeled ascent rate, hereby indicating a probable downward air motion. On the contrary, it is more likely that the overestimation of the ascent rate below $2 \mathrm{~km}$ altitude is due to the inaccuracy of the (corrected) reference drag curve. It should be mentioned that the presence of an unwinder between the balloon and its payload during the actual flight can be held responsible for part of the overestimation by the model. The unwinder - whose role is to progressively increase the length of the cable linking the payload 

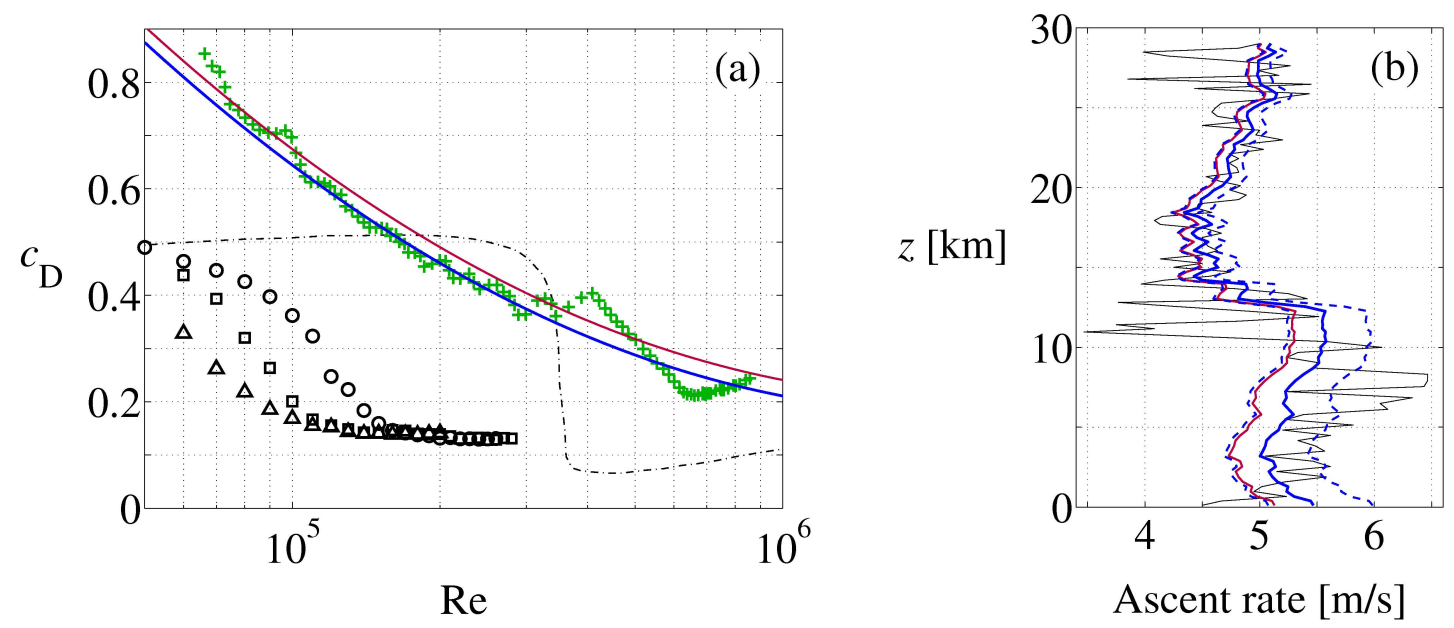

Fig. 6. Evaluation of the model on LUAMI flight L003b launched on 5 November 2008 at 22:45 UTC. (a) Corrected reference drag curve (-) obtained by shifting the reference drag curve (-, see Fig. 3) by -0.03 along the $c_{\mathrm{D}}$-axis. The experimental drag curve derived from the flight is indicated by the green crosses. The curves by Achenbach (1972) and Son et al. (2010) for a perfect sphere are reported here for comparison (see Fig. 1). (b) Vertical profile of the balloon ascent rate in still air derived from the corrected drag curve (-), and the lower and upper limits of its range of uncertainty (---). The ascent rate in still air derived from the non-corrected reference drag curve (solid purple curve in panel (a)) is indicated here for comparison (-), along with the 60 s-low pass filtered ascent rate calculated from the GPS data (-).

to the balloon - remains active during the first 60 to $120 \mathrm{~s}$ of flight. Since the final length of the cable is about $50 \mathrm{~m}$, this implies that the unwinder reduces the ascent rate of the payload as compared to that of balloon by 0.5 to $1 \mathrm{~m} \mathrm{~s}^{-1}$ in the lowermost 300 to $600 \mathrm{~m}$ of the ascent, which explains the lowermost part of the discrepancy between the modeled and the measured vertical velocities. No sharp conclusion can however be drawn regarding the precision of the model since the air vertical velocity was not measured independently during the LUAMI campaign.

The range of uncertainty in the ascent rate profile is obtained from the two additional runs of the model based on the reference drag curve shifted by $+\sigma_{c_{\mathrm{D}}}^{*}$ and $-\sigma_{c_{\mathrm{D}}}^{*}$ along the $c_{\mathrm{D}^{-}}$ axis, respectively, where $\sigma_{c_{\mathrm{D}}}^{*}$ denotes the standard deviation of the difference between the corrected reference drag curve and the experimental drag curve (see end of Sect. 3). In the case of the example pictured in Fig. $6, \sigma_{c_{\mathrm{D}}}^{*}=0.03$. The corresponding uncertainty in $v_{z}$ is shown in panel (b) of the figure; it is observed to decrease significantly when crossing the tropopause $(z=12 \mathrm{~km})$ while remaining globally constant over the troposphere and the stratosphere separately. This suggests the use of two different uncertainty ranges, the first one associated with the troposphere and the second one with the stratosphere. Averaging the uncertainty in $v_{z}$ below and above the tropopause, respectively, it is found that the balloon ascent rate in still air is defined up to an additive factor of $\pm 0.4 \mathrm{~m} \mathrm{~s}^{-1}$ in the troposphere, while this factor reduces to $\pm 0.2 \mathrm{~m} \mathrm{~s}^{-1}$ in the stratosphere. The uncertainty error in $v_{z}$ therefore decreases by a factor of $\sim 2$ when crossing the tropopause.
Evaluation of the model on the nine remaining LUAMI flights results in observations similar to those described above. The uncertainty in the modeled ascent rate averaged over the whole dataset is $\sim 0.5 \mathrm{~m} \mathrm{~s}^{-1}$ in the troposphere and $\sim 0.2 \mathrm{~m} \mathrm{~s}^{-1}$ in the stratosphere. As a consequence, it is assumed that the present model calculates the balloon ascent rate in still air with uncertainties of $\pm 0.5 \mathrm{~m} \mathrm{~s}^{-1}$ and $\pm 0.2 \mathrm{~m} \mathrm{~s}^{-1}$ below and above the tropopause, respectively, in the case where the flight data can be used to correct the reference drag curve. In comparison, Wang et al. (2009) model the balloon ascent rate in still air with an uncertainty of $\pm 0.9 \mathrm{~m} \mathrm{~s}^{-1}$. On top of its increased accuracy, the present model enables the fairly good derivation of the ascent rate below $5 \mathrm{~km}$ altitude, contrary to the model by Wang et al. which systematically underestimates the ascent rate in this altitude range. As an example, a comparison of the two models on a particular flight is pictured in Fig. 7a. The present model is observed to be in greater agreement with the smoothed observations, particularly in the troposphere $(z<12 \mathrm{~km})$. This results in the altitude of the balloon as a function of time being modeled more accurately, as shown in Fig. 7b.

In the case where the flight data are not available to correct the reference drag curve (e.g. in forecasting applications), the uncertainty in the latter is higher; in particular, its associated values of the drag coefficient are determined up to a precision of $\pm \sigma_{c_{\mathrm{D}}}= \pm 0.04$ (see Sect. 2.3). Similarly to above, the corresponding uncertainty in the modeled ascent rate is obtained by computing the difference between the profile derived by the first run of the model and the two additional profiles based on the reference drag curve shifted by $+\sigma_{c_{\mathrm{D}}}$ and $-\sigma_{c_{\mathrm{D}}}$ along the $c_{\mathrm{D}}$-axis, respectively. The average 

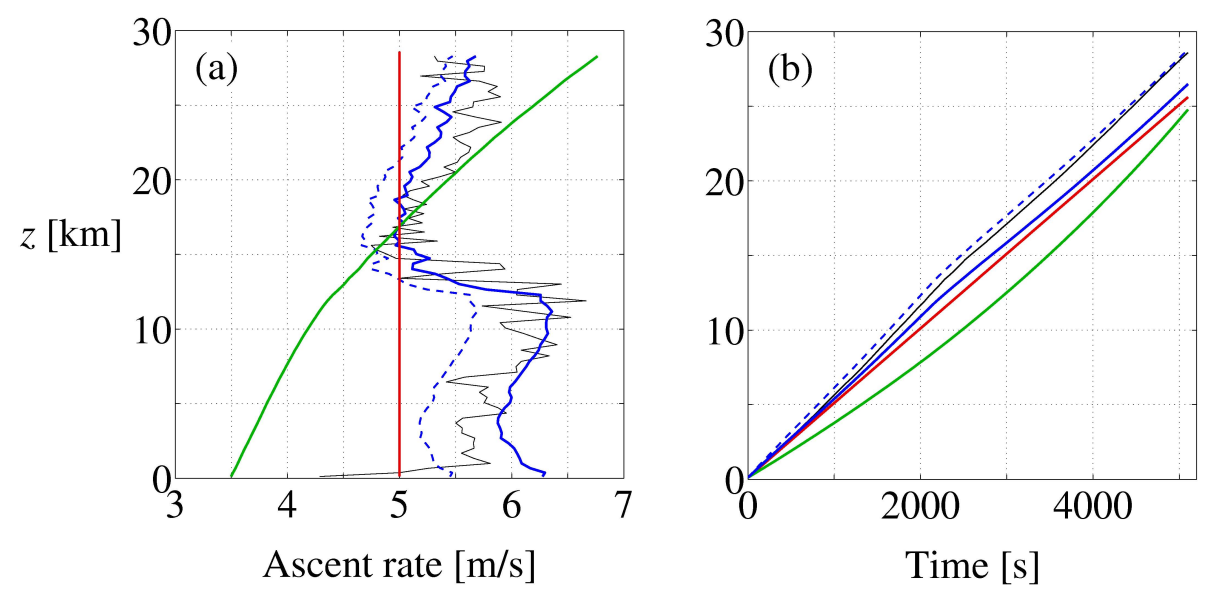

Fig. 7. Comparison of the predictions by different models with data measured during the balloon ascent in the case of LUAMI flight L005 launched on 6 November 2008 at 22:45 UTC. Measured data (-); predictions by the present model based either on the shifted reference drag curve (-) or on the reference drag curve itself (---); predictions by the model by Wang et al. (2009) (-); predictions by the model by Engel (2009) (-). (a) Vertical profile of the balloon ascent rate. (b) Altitude of the balloon as a function of time.

over the ten LUAMI flights estimates the uncertainty in the modeled ascent rate to be $\pm 0.6 \mathrm{~m} \mathrm{~s}^{-1}$ in the troposphere and $\pm 0.3 \mathrm{~m} \mathrm{~s}^{-1}$ in the stratosphere in this case. These uncertainty ranges are slightly larger than in the case where the reference drag curve can be corrected; they however remain smaller than those of the model by Wang et al. (2009). As pictured in Fig. 7a, the absence of correction to the reference drag curve may result in a systematic offset of the most probable ascent rate derived from the first run of the model as compared to the measured ascent rate. This is thought to result from differences in the manufacturing process of the individual balloons, responsible for an unpredictable variation of the drag coefficient from one balloon to the other, as mentioned previously in Sect. 2.3. In practice, this implies that the present model may systematically over- or underestimate the balloon altitude as a function of time when used to forecast the balloon trajectory, as can be observed for example in Fig. 7b. The magnitude of the systematic error in the modeled ascent rate is bounded by the aforementioned limit of the uncertainty in $v_{z}$, namely $0.6 \mathrm{~m} \mathrm{~s}^{-1}$ in the troposphere and $0.3 \mathrm{~m} \mathrm{~s}^{-1}$ in the stratosphere. It should be mentioned that the current accuracy of the drag coefficient is closely linked to the LUAMI flight data set used for the derivation of the drag curve. Extending this analysis to more soundings with carefully recorded payload and uplift masses is therefore highly desirable.

The present model based on the (non-corrected) reference drag curve proves a better forecasting tool than the one by Engel (2009), which assumes for simplicity a constant ascent rate of $5 \mathrm{~m} \mathrm{~s}^{-1}$. As a matter of fact, the error in the calculated balloon altitude at burst time, averaged over the ten LUAMI flights, is $1.4 \mathrm{~km}$ when using the present model as opposed to $2.7 \mathrm{~km}$ when using the model by Engel (not shown). The predictions of the two models can be compared on the par- ticular example of Fig. 7a. It is observed that, despite its systematic offset, the present model based on the reference drag curve matches more precisely the overall profile of the measured ascent rate. This results in the altitude of the balloon as a function of time being forecasted more accurately by the present model, as shown in Fig. $7 \mathrm{~b}$.

\subsection{Derivation of the vertical air motion}

Given the above evidence for the model accuracy, the present section aims at illustrating an application: vertical air motion is estimated from the data collected during LUAMI flight L003a launched on 11 November, at 22:45 UTC. To this end, the balloon ascent rate in still air is calculated according to the model and then subtracted from the measured balloon ascent rate, as pictured in Fig. 8. The resulting profile of the air vertical velocity shown in panel (b) is difficult to validate owing to the same limitation as already encountered by Wang et al. (2009), namely the "lack of coincident [vertical velocity] data from other measurements." In an attempt at compensating for this lack, the potential temperature lapse rate measured during the flight is taken as an approximate proxy for the vertical velocity. Indeed, in a first approximation, air parcels advected upwards cool down adiabatically on small spatial scales. As a consequence, their potential temperature, $\theta_{\mathrm{a}}$, remains approximately constant on such scales. We therefore expect the vertical profile of the potential temperature lapse rate, $\mathrm{d} \theta_{\mathrm{a}} / \mathrm{d} z$, to present sharp decreases in regions of vertical updraft. Conversely, we expect the potential temperature lapse rate to increase significantly in regions of vertical downdraft, where air parcels of higher altitude and with larger potential temperature are advected downwards. Thus, in a first approximation, the profiles of the estimated vertical velocity of air and the potential temperature lapse rate should 


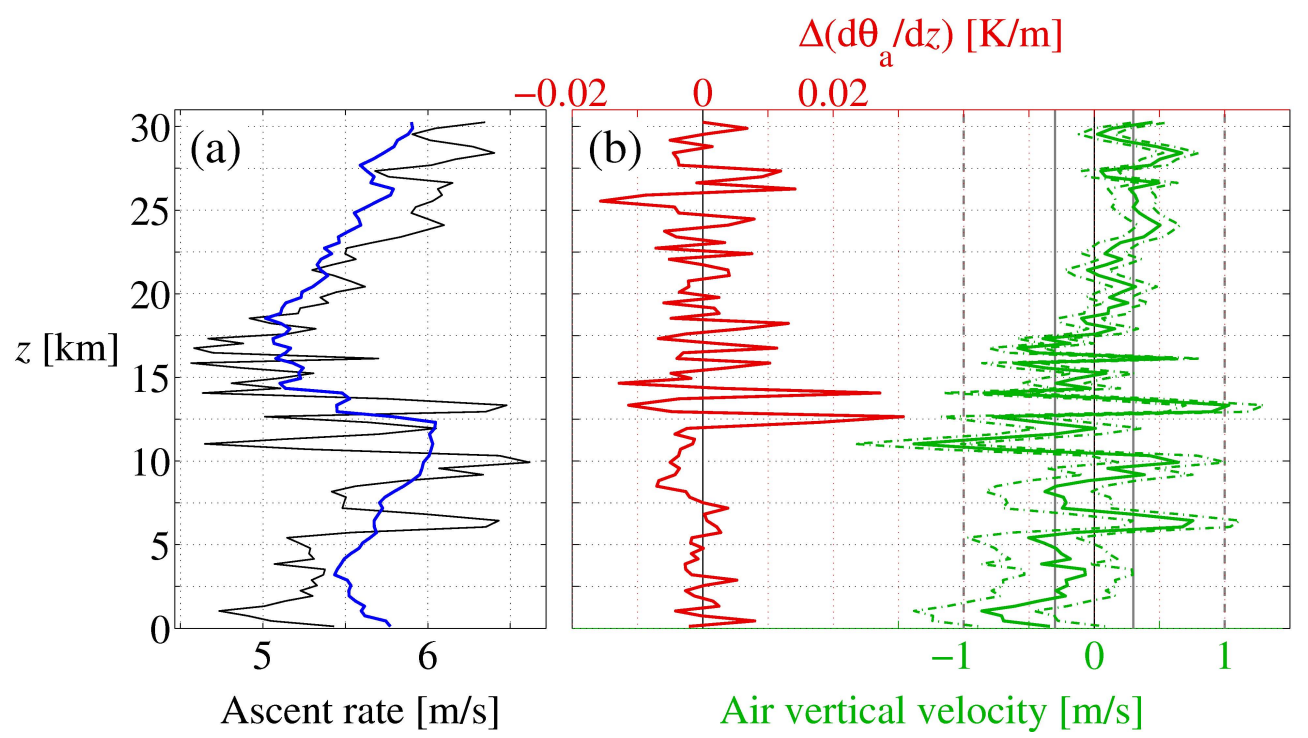

Fig. 8. Air vertical velocity during LUAMI flight L003a launched on 5 November 2008 at 22:45 UTC. (a) Balloon ascent rate in still air as calculated from the model (-); actual balloon ascent rate derived from the GPS data (-). (b) Air vertical velocity obtained by subtracting the ascent rate in still air from the actual ascent rate $(-)$, and the upper and lower limits of its associated range of uncertainty $(-\cdot-\cdot-)$; deviations of the potential temperature lapse rate from its still air value, derived from the atmospheric temperature recorded during the balloon ascent (-). The vertical velocities derived by Hoyle et al. (2005) from aircraft measurements are indicated here as thin gray lines for comparison: typical gravity-wave fluctuations, $\pm 0.3 \mathrm{~m} \mathrm{~s}^{-1}(-)$; strong fluctuations representing less than $\sim 2 \%$ of all wave occurrences, $\pm 1 \mathrm{~m} \mathrm{~s}^{-1}(---)$.

present evidences of anti-correlation. This reasoning is nevertheless limited, since temperature fluctuations can be sensitive to both low- and high-frequency gravity waves, whereas vertical velocity fluctuations are more affected by higherfrequency gravity waves (Lane et al., 2003; Geller and Gong, 2010). As such, Gong and Geller (2010) experimentally observe that "the apparent dominant vertical wavelengths [of the gravity waves] estimated from $T^{\prime}$ [(temperature fluctuations)] and $w^{\prime}$ [(vertical velocity fluctuations)] profiles are different for some cases."

Evidences of anti-correlation are however apparent on Fig. $8 \mathrm{~b}$, which pictures the vertical profile of $\Delta\left(\mathrm{d} \theta_{\mathrm{a}} / \mathrm{d} z\right)$ beside the estimated profile of the air vertical velocity. The quantity $\Delta\left(\mathrm{d} \theta_{\mathrm{a}} / \mathrm{d} z\right)$ corresponds here to the potential temperature lapse rate from which its mean value over the troposphere or stratosphere, depending on the altitude at which it is evaluated, has been subtracted. A particularly noticeable example of anti-correlation can be found in the altitude range $12-15 \mathrm{~km}$, where the fluctuation amplitudes of the air vertical velocity and of the potential temperature lapse rate are relatively large. The correlation coefficient between the two profiles is -0.31 , and the probability that this value could be obtained at random from two independent distributions is as low as $2.4 \times 10^{-3}$. This suggests that the profiles of the air vertical velocity and of $\Delta\left(\mathrm{d} \theta_{\mathrm{a}} / \mathrm{d} z\right)$ are globally anticorrelated.

However, the sole comparison with the potential temperature lapse rate does not enable us to validate the estimated vertical air motion owing to the aforementioned limitations. This comparison also does not provide any quantitative information on the precision of the derived air vertical velocity. The analysis of the model uncertainty in the previous section however suggests that the uncertainty error of this velocity is within the range $\pm 0.5 \mathrm{~m} \mathrm{~s}^{-1}$ in the troposphere and $\pm 0.2 \mathrm{~m} \mathrm{~s}^{-1}$ in the stratosphere, as indicated in panel (b) of Fig. 8. Moreover, the estimated velocity is within the range of the typical vertical wind fluctuations in the troposphere reported by Hoyle et al. (2005) and indicated as thin gray lines in Fig. 8b. These fluctuations were derived from aircraft measurements performed during the SUCCESS campaign (Subsonic Aircraft: Contrail and Cloud Effects Special Study) which took place in the middle troposphere in cirrus clouds over the eastern Pacific Ocean. In their derivations, Hoyle et al. (2005) made sure to avoid perturbated regions to focus on free tropospheric gravity waves, similar to the situation during the LUAMI campaign in the northern German flatland.

One may argue that the vertical air motion could be estimated by a much more simplistic approach than the one presented above. Indeed, to obtain an approximation of the balloon ascent rate in still air, one may simply consider the smoothed profile of the actual balloon ascent rate (see Sect. 2.3) instead of using the balloon ascent model. A comparison of this simplistic approach with the one based on the model is shown in Fig. 9 in the case of LUAMI flight L025. The respective profiles of the balloon ascent rate in still air 

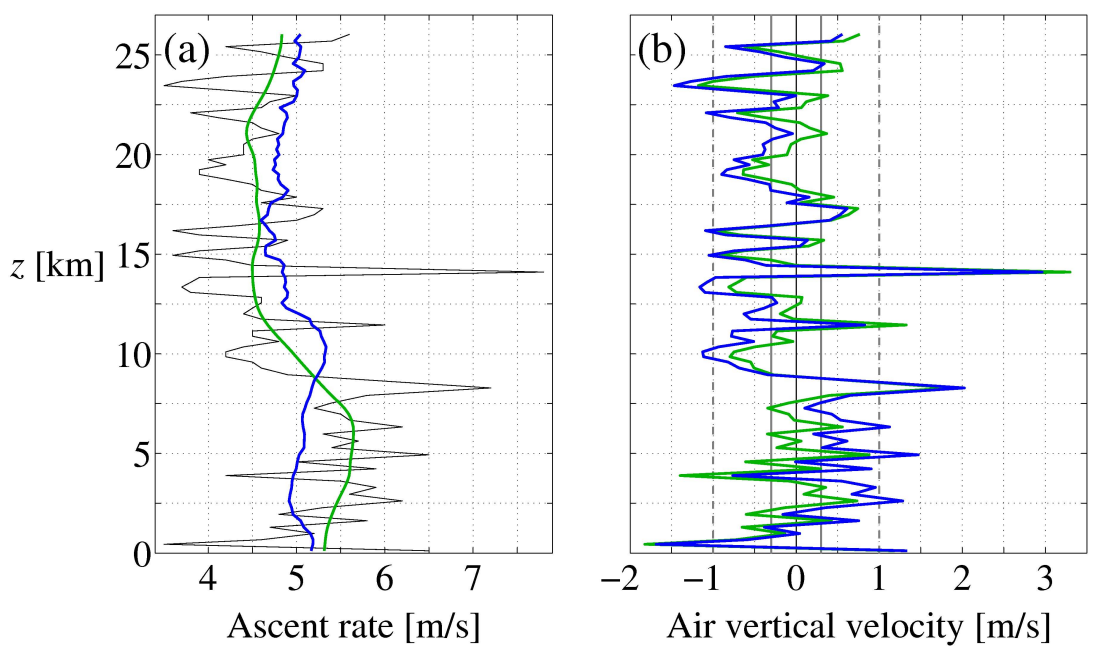

Fig. 9. Comparison of the model with the method based on the smoothing of the measured balloon ascent rate in the case of LUAMI flight L025 launched on 19 November 2008 at 22:45 UTC. (a) Vertical profile of the balloon ascent rate in still air derived from the model (-); smoothed profile of the balloon ascent rate measured during the actual flight (-) (for a description of the smoothing technique, see Sect. 2.3). The actual ascent rate derived from the GPS data is indicated as a thin black line for comparison. (b) Corresponding profiles of the air vertical velocity estimated from the model $(-)$ and from the smoothed profile of the measured balloon ascent rate (-). The vertical velocities derived by Hoyle et al. (2005) from aircraft measurements are indicated here as thin gray lines for comparison: typical gravity-wave fluctuations, $\pm 0.3 \mathrm{~m} \mathrm{~s}^{-1}(-)$; strong fluctuations representing less than $\sim 2 \%$ of all wave occurrences, $\pm 1 \mathrm{~m} \mathrm{~s}^{-1}$ (---).

estimated by the two methods are relatively dissimilar (see panel (a)). The one derived from the method using the model presents a finer resolution: it responds more physically to the fluctuations of the atmospheric temperature. In panel (b) of Fig. 9, it can be observed that the respective estimations of the air vertical velocity by the two methods differ by up to $0.5 \mathrm{~m} \mathrm{~s}^{-1}$ either in the troposphere and in the stratosphere. Yet, the method based on the model cannot be proven to describe the balloon ascent more precisely than the other one. The absence of independent measurements of the vertical air motion during the LUAMI campaign make the quantitative evaluation of any of the two approaches impossible.

\section{Discussion and conclusion}

Very few models of the ascent of sounding balloons in the atmosphere are available to date (Engel, 2009; Wang et al., 2009). In this study, a new model is proposed and shown to be an improvement over the present state of the art. Derived by equating the free lift and the drag force, the balloon ascent rate in still air is found to depend on three variables: the air mass density, the balloon drag coefficient and the balloon effective radius. The air mass density is assumed to be known either from numerical weather forecast or from the atmospheric temperature and pressure measured during the flight. The balloon effective radius, defined as the radius of the balloon's volume-equivalent sphere, is computed at each step of the model in three stages: (i) the balloon is first adiabatically expanded; (ii) heat is then allowed to diffuse at con- stant pressure from the surrounding air into the balloon while assuming the lifting gas to be incompressible; and (iii) the effective radius and temperature distribution of the balloon are finally corrected to account for the expansion of the lifting gas discarded in step (ii). Since solar radiation - which has a strong impact on the balloon temperature distribution - is not resolved, the model is only applicable to night flights in its present state. Application to daytime soundings calls for a further study, but it should be possible provided that solar radiation is modeled as a diffusive process inside the balloon and that heating of the balloon envelope is taken into account. To compensate for the lack of data on the drag coefficient of almost spherical objects in a turbulent medium, a reference drag curve for sounding balloons is derived from a dataset of flights launched during the LUAMI campaign. This drag curve applies only to a particular type of sounding balloon, but using the methods we describe in this paper, it should be straightforward to derive a similar curve for other types of balloon. At each step of the model, the balloon drag coefficient can be obtained from the reference drag curve by refining the initial estimate of the Reynolds number through a loop.

A priori, the ascent rate in still air predicted by the model has an uncertainty of $\pm 0.6 \mathrm{~m} \mathrm{~s}^{-1}$ in the troposphere and $\pm 0.3 \mathrm{~m} \mathrm{~s}^{-1}$ in the stratosphere, where the range of uncertainty is defined as a difference of plus or minus one standard deviation from the calculated value. For some flights, a systematic offset between the predictions of the model and the subsequently measured actual ascent rate points to differences in the manufacturing process of the individual 

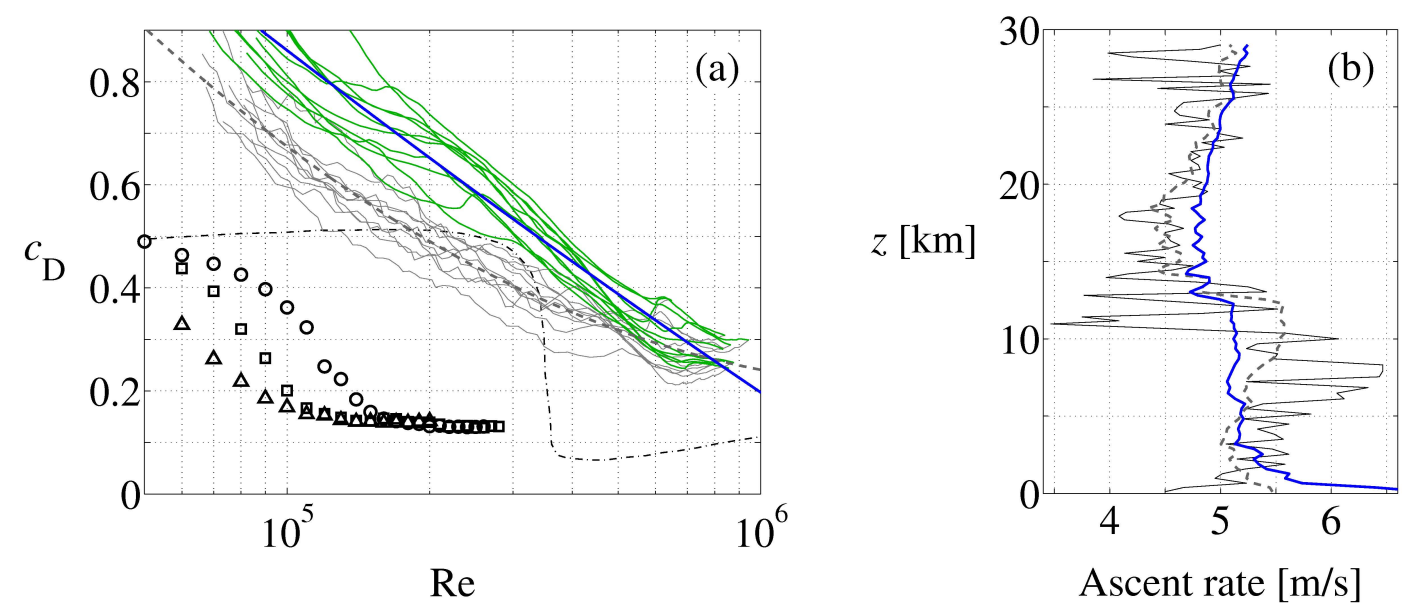

Fig. 10. Effect of the ten-fold increase of the mean molecular heat diffusion coefficient on the model. (a) Experimental drag curves derived from the ten LUAMI flights (-), and their associated reference drag curve (-), in the case of the enhanced $\langle D\rangle$. The ten experimental curves $(-)$ and the reference drag curve (-- ) pictured in Fig. 3 are reported here for comparison, along with the curves by Achenbach (1972) and Son et al. (2010) for a perfect sphere. (b) Vertical profile of the balloon ascent rate in still air calculated from the corrected reference drag curve in the case of LUAMI flight L003b (see Fig. 6); $\langle D\rangle$ increased by a factor of ten (-); $\langle D\rangle$ normal (---). The 60-s low pass filtered vertical profile of the ascent rate calculated from the GPS data is indicated here for comparison (-).

balloons. These differences are responsible for unpredictable departures of the balloon drag coefficient from the reference drag curve and result in a mean uncertainty error of $\pm 1.5 \mathrm{~km}$ in the altitude of the balloon at burst time predicted by the model. The curve of the ascent rate in still air as a function of altitude captures the measured ascent rate profile very well, suggesting the model to be a valuable a priori trajectory forecast tool. As such, the algorithm could be used, for example, to improve the precision of the balloon trajectory forecasts required during match flight campaigns. Up to the present, forecast trajectory models used during such campaigns have assumed a constant ascent rate of $5 \mathrm{~m} \mathrm{~s}^{-1}$ for the balloon (e.g. Engel, 2009).

A posteriori, the data collected during the ascent can be used to adapt the reference drag curve and hereby reduce the discrepancy between the modeled and measured ascent rate profiles, as described in the final paragraph of Sect. 3. In this case, the air vertical velocity can be evaluated by subtracting the ascent rate in still air from the actual ascent rate. This procedure is shown to provide an estimation of the air motion which is within the range of the typical air velocity fluctuations derived by Hoyle et al. (2005) from the SUCCESS campaign in the middle troposphere (see panel (b) of Figs. 8 and 9). Its uncertainty error is estimated to be $0.5 \mathrm{~m} \mathrm{~s}^{-1}$ in the troposphere and $0.2 \mathrm{~m} \mathrm{~s}^{-1}$ in the stratosphere. In case this uncertainty could be reduced, the air vertical velocity derived in this way would be useful, for example to parametrize the cooling rate in cirrus cloud box models (Hoyle et al., 2005).

The neglect of heat eddy diffusion or heat convection inside the balloon affects the reference drag curve and the accuracy of the model. Indeed, assuming eddy diffusion or convection leads to an enhanced transfer of heat into the balloon and therefore to an increase of the expansion of the balloon volume with altitude. As a consequence, the uplift force is larger mainly in the stratosphere, where the influence of the heat transfer into the balloon on the ascent rate is the strongest. This results - mainly in the region corresponding to the stratosphere $\left(5 \times 10^{4} \leqslant R e \leqslant 5 \times 10^{5}\right)$ - in the increase of the experimental drag curves derived from the ten LUAMI flights, as pictured in Fig. 10a, where $\langle D\rangle$ has been increased by a factor of ten in order to simulate eddy diffusion. As observed in the figure, the reference drag curve is steeper and shifted upwards in the case where eddy diffusion is resolved as compared to the case where only molecular diffusion is assumed. Based on this curve and the molecular heat diffusion coefficient increased ten times, the model is found to not capture the general feature of the ascent rate profile and particularly the maximum close to the tropopause. This appears clearly in the example pictured in panel (b) of Fig. 10, where the vertical profiles of $v_{z}$ obtained from the model based on $\langle D\rangle$ and $10\langle D\rangle$, respectively, can be compared. This suggests that heat eddy diffusion and heat convection are not likely and that the main process responsible for the propagation of heat inside the balloon is molecular diffusion.

The model can be improved with respect to several aspects. Firstly, more experimental night flights should be used for the derivation of the reference drag curve, also during other seasons and in other locations. This would give the statistical mean performed by the polynomial fit more relevance from an ensemble point of view. Only ten flights are considered in this study owing for the plain difficulty to find high resolution datasets including accurate measurements of the uplift and payload masses. Indeed, as already noted by Wang et al. (2009), the uplift and payload masses 
are currently neither measured precisely nor stored systematically before each flight. In fact, the information regarding these masses could be found only in the case of the balloons launched during the LUAMI campaign. Unfortunately, even during the LUAMI campaign it was not considered that mass measurements of great precision would be required later, which explains a part of the spread of the experimental drag curves discussed in Sect. 2.3. We therefore strongly suggest that the balloon launch protocols must take account of precise measurements and recordings of both the payload and uplift masses. Secondly, radiative heat transfer into the balloon could be resolved, which would allow for day flights to be modeled. Taking solar radiation into account would require the balloon envelope emissivity and the cloud cover to be considered, which would substantially complicate the treatment of heat inside the balloon. Finally, the validation of the reference drag curve lacks the support of studies on the drag coefficient of sounding balloons. In particular, the mechanisms at the origin of the large magnitude of this drag coefficient should be investigated in more detail. This includes an analysis of the deformation of the balloon shape during the ascent and a better characterization of both the lift-induced drag and the drag coefficient of almost spherical objects at very high Reynolds numbers and non-negligible turbulence intensity levels. Independent measurements of the air vertical velocity would also be useful for the validation of the reference drag curve.

\section{Appendix A}

\section{Derivation of the characteristic time of diffusion}

The analytical solution to Eq. (4) provided with the boundary conditions $T_{\mathrm{b}}(1, t)=T_{\mathrm{a}}(t)$ and $\left(\partial T_{\mathrm{b}} / \partial r\right)_{r=0}=0$ for all $t>0$ reads (Carslaw and Jaeger, 1959):

$$
T_{\mathrm{b}}(r, t)=\frac{2}{r} \sum_{n=1}^{\infty}\left(\alpha_{n}+\beta_{n}(t)\right) e^{-D(\pi n / R)^{2} t} \sin (\pi n r),
$$

where

$$
\begin{aligned}
& \alpha_{n}=\int_{0}^{1} r T_{\mathrm{b}, 0}(r) \sin (\pi n r) \mathrm{d} r, \\
& \beta_{n}(t)=\frac{\pi D}{R^{2}} n(-1)^{n+1} \int_{0}^{t} T_{\mathrm{a}}(s) e^{D(\pi n / R)^{2} s} \mathrm{~d} s,
\end{aligned}
$$

and $T_{\mathrm{b}, 0}:[0,1] \mapsto \mathbb{R}$ denotes the initial temperature distribution. In Eq. (A1), $r \in[0,1]$ and $t \geqslant 0$. The solution adopts a much simpler expression in the case where the initial temperature distribution is uniform, viz. $T_{\mathrm{b}, 0}$ is a constant, and the temperature at $r=1$ is kept constant over time, viz. $T_{\mathrm{a}}$ is constant. In such a case,

$$
\frac{T_{\mathrm{a}}-T_{\mathrm{b}}(r, t)}{T_{\mathrm{a}}-T_{\mathrm{b}, 0}}=\frac{2}{\pi r} \sum_{n=1}^{\infty} \frac{(-1)^{n+1}}{n} e^{-D(\pi n / R)^{2} t} \sin (\pi n r),
$$

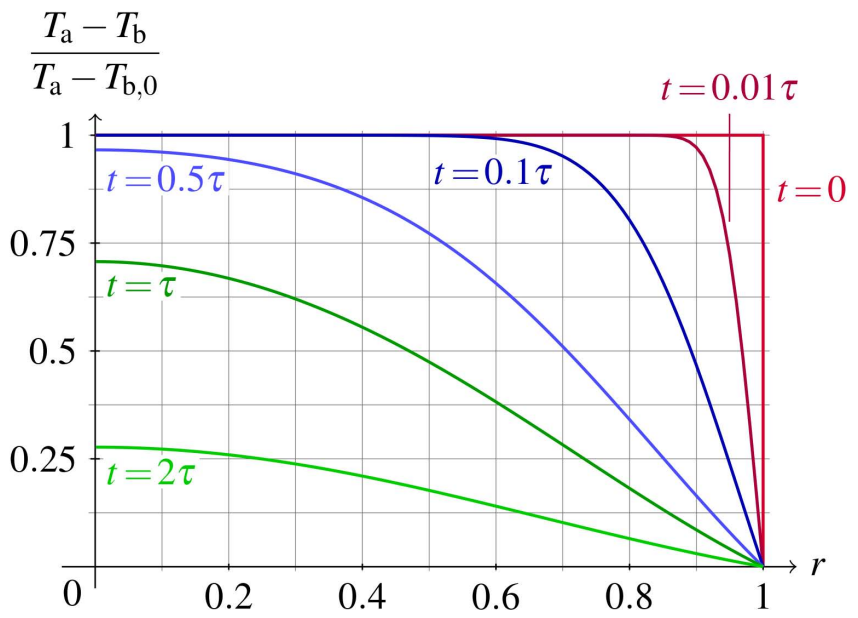

Fig. A1. Radial distribution of the quantity on the left-hand side of Eq. (A2) at different times. $\tau=R^{2} /\left(\pi^{2} D\right)$ denotes the characteristic time of diffusion.

Table A1. Typical values of some parameters associated with the balloon at two different altitudes. The lifting gas is assumed to be hydrogen, whose specific heat capacity at constant pressure equals $1.4 \times 10^{5} \mathrm{~J} \mathrm{~kg}^{-1} \mathrm{~K}^{-1}$ ).

\begin{tabular}{lccc}
\hline Altitude & $R(\mathrm{~m})$ & $\left.\left.\kappa \mathrm{W} \mathrm{m}^{-1} \mathrm{~K}^{-1}\right)\right)$ & $\rho_{\mathrm{b}}\left(\mathrm{kg} \mathrm{m}^{-3}\right)$ \\
\hline ground & 1 & 0.18 & 0.09 \\
$30 \mathrm{~km}$ & 4 & 0.14 & $10^{-3}$ \\
\hline
\end{tabular}

where the quantity on the left-hand side is the temperature difference between the outside and the inside of the balloon normalized by the initial difference. The radial profile of this quantity is shown in Fig. A1 for different times. The characteristic time of diffusion is obtained from Eq. (A2) by considering only the dominant coefficient associated to $n=1$ in the Fourier series, which leads to $\tau=R^{2} /\left(\pi^{2} D\right)$. Using the expression $D=\kappa /\left(\rho_{\mathrm{b}} c_{p}\right)$ and the typical values of Table A1, the characteristic time of diffusion is observed to decrease from $\sim 900 \mathrm{~s}$ at ground to $\sim 300 \mathrm{~s}$ at $30 \mathrm{~km}$ altitude in the case where the lifting gas is hydrogen. Diffusion occurs faster at higher altitude as a result of the lower mass density of the lifting gas.

\section{Appendix B}

\section{Convergence study of the finite element code}

In the balloon ascent model, Eq. (4) is discretized spatially according to the Finite Element Method. The numerical solution is expressed in terms of a basis of second-order polynomials, which corresponds to a discretization scheme of second order in space. Regarding the time discretization, the first-order Euler backwards scheme is preferred - for stability 


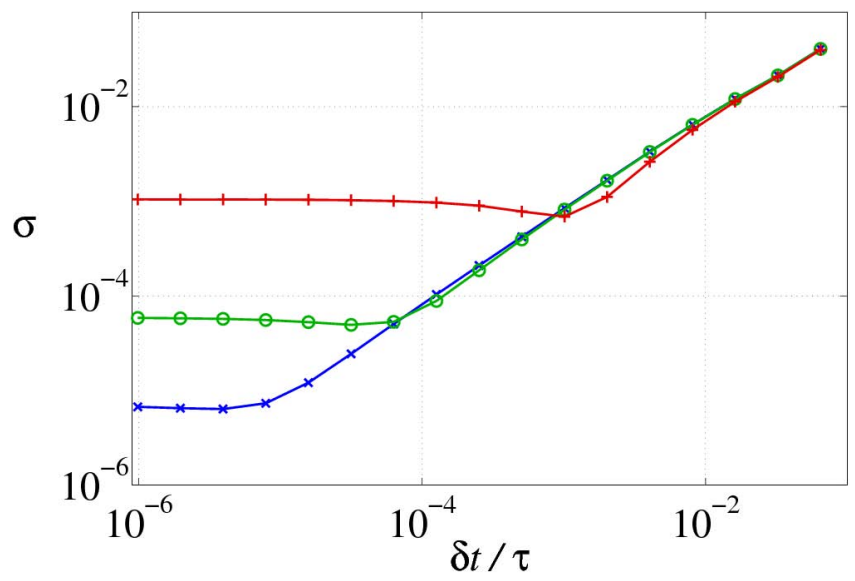

Fig. B1. Variation of $\sigma$ at $t=0.1 \tau$ as a function of the time step. Three different space discretization intervals are considered: $\delta r=$ $10^{-2}(*) ; \delta r=2 \times 10^{-2}(\theta)$; and $\delta r=5 \times 10^{-2}(\vdash)$

purposes - to the second-order Cranck-Nicolson one. The latter introduces oscillations in the numerical solution when used in association with the Finite Element Method.

Convergence of the numerical solution is analyzed here in the simple reference case where the initial temperature inside the balloon is uniform and the temperature at the balloon's surface is constant. Let $T_{\mathrm{b} \text {, ana }}$ and $T_{\mathrm{b} \text {,num }}$ respectively denote the analytical and numerical solutions in this case, the expression of $T_{\mathrm{b} \text {, ana }}$ being derived from Eq. (A2). Convergence is measured in terms of the second moment of the difference $T_{\mathrm{b}, \text { ana }}-T_{\mathrm{b}, \text { num }}$,

$\sigma^{2}(t)=\frac{3}{\left(T_{\mathrm{a}}-T_{\mathrm{b}, 0}\right)^{2}} \int_{0}^{1} r^{2}\left[T_{\mathrm{b}, \text { ana }}(r, t)-T_{\mathrm{b}, \text { num }}(r, t)\right]^{2} \mathrm{~d} r$,

where $T_{\mathrm{a}}$ and $T_{\mathrm{b}, 0}$ are defined as in Appendix A. The quantity $\sigma$ corresponds to the numerical error averaged over the balloon volume and normalized by the initial temperature difference between the inside and the outside of the balloon.

Let $\delta r$ and $\delta t$ respectively denote the space discretization interval and the time step used by the numerical scheme. Variation of $\sigma$ at a fixed time as a function of $\delta t$ is shown in Fig. B1 for three different values of $\delta r$. It is observed that the numerical error scales linearly with the time step and saturates for small values of $\delta t$. The error does not depend on $\delta r$ for large time steps, contrary to the saturation value. This implies that a finer spatial discretization is valuable only if conjugated with a finer time resolution. In practice, a time step of $10^{-3} \tau$ is chosen in the balloon ascent model, as it is observed to result in relatively short computational times (not shown) while leading to an acceptable mean error of $0.1 \%$ compared to the analytical solution. This implies that a space discretization as large as $5 \times 10^{-2}$ can be used, as a finer choice of $\delta r$ would not improve the precision of the numerical solution (see Fig. B1).
Acknowledgements. We gratefully acknowledge the LUAMI team for its careful work, without which this study would have been much more intricate. We also thank Hendrick Huwald at École Polytechnique Fédérale de Lausanne (EPFL, Lausanne, Switzerland) for fruitful discussions and his revision of our work. This work was in part supported within the COST action ES0904, Atmospheric Water Vapour in the Climate System, funded by the Swiss State Secretariat for Education and Research (SER).

Edited by: A. Stoffelen

\section{References}

Achenbach, E.: Experiments on the flow past spheres at very high Reynolds numbers, J. Fluid Mech., 54, 565-575, 1972.

Alexander, M., Geller, M., McLandress, C., Polavarapu, S., Preusse, P., Sassi, F., Sato, K., Eckermann, S., Ern, M., Hertzog, A., Kawatani, Y., Pulido, M., Shaw, T. A., Sigmond, M., Vincent, R., and Watanabe, S.: Recent developments in gravitywave effects in climate models and the global distribution of gravity-wave momentum flux from observations and models, Q. J. Roy. Meteorol. Soc., 136, 1103-1124, doi:10.1002/qj.637, 2010.

Carslaw, H. and Jaeger, J.: Conduction of Heat in Solids, Clarendon Press, 2nd Edn., 1959.

Engel, I.: Trajectory Modelling of Match Balloon Soundings for Cirrus Cloud Characterisation, Master's thesis, Swiss Federal Institute of Technology Zurich, 2009.

Fritts, D. and Alexander, M.: Gravity wave dynamics and effects in the middle atmosphere, Rev. Geophys., 41, 1003, doi:10.1029/2001RG000106, 2003.

Geller, M. A. and Gong, J.: Gravity wave kinetic, potential, and vertical fluctuation energies as indicators of different frequency gravity waves, J. Geophys. Res., 115, D11111, doi:10.1029/2009JD012266, 2010.

Gong, J. and Geller, M. A.: Vertical fluctuation energy in United States high vertical resolution radiosonde data as an indicator of convective gravity wave sources, J. Geophys. Res., 115, D11110, doi:10.1029/2009JD012265, 2010.

Govardhan, R. and Williamson, C.: Vortex-induced vibrations of a sphere, J. Fluid Mech., 531, 11-47, doi:10.1017/S0022112005003757, 2005.

Hamilton, K. and Vincent, R.: High-resolution radiosonde data offer new prospects for research, EOS Transactions, 76, 497, doi:10.1029/95EO00308, 1995.

Hoyle, C., Luo, B., and Peter, T.: The origin of high ice crystal number densities in cirrus clouds, J. Atmos. Sci., 62, 2568-2579, 2005.

Immler, F.: Report on the LUAMI campaign, Tech. Rep., WMO, available at: http://www.hydrometeoindustry.org/ Meetings2008/LUAMI_Lindengerg_Nov2008/LUAMI_v2.pdf, last access: Fall 2008, 2008.

Lane, T. P., Reeder, M. J. and Guest, F. M.: Convectively generated gravity waves observed from radiosonde data taken during MCTEX, Q. J. Roy. Meteorol. Soc., 129, 1731-1740, doi:10.1256/qj.02.196, 2003.

Lewis, R., Morgan, K., Thomas, H., and Seetharamu, K.: The Finite Element Method in Heat Transfer Analysis, John Wiley \& Sons Inc, 1996. 
Loth, E.: Drag of non-spherical solid particles of regular and irregular shape, Powder Technol., 182, 342-353, 2008.

Mapleson, W.: The drag of spherical rubber balloons, Q. J. Roy. Meteorol. Soc., 80, 449-451, 1954.

Musso, I., Cardillo, A., Cosentino, O., and Memmo, A.: A balloon trajectory prediction system, Adv. Space Res., 33, 1722-1726, doi:10.1016/j.asr.2003.07.044, 2004.

Neve, R.: The importance of turbulence macroscale in determining the drag coefficient of spheres, Int. J. Heat Fluid Fl., 7, 28-36, 1986.

Palumbo, R.: A Simulation Model for Trajectory Forecast, Performance Analysis and Aerospace Mission Planning with High Altitude Zero Pressure Balloons, Ph.D. thesis, Università degli Studi di Napoli "Federico II", available at: http://www.fedoa.unina.it/1839/1/Palumbo_Ingegneria Aerospaziale_Navale_e_della_Qualita.pdf, 2007.

Rex, M., Von Der Gathen, P., Braathen, G., Harris, N., Reimer, E., Beck, A., Alfier, R., Krüger-Carstensen, R., Chipperfield, M., De Backer, H., Balis, D., O'Connor, F., Dier, H., Dorokhov, V., Fast, H., Gamma, A., Gil, M., Kyrö, E., Litynska, Z., Mikkelsen, I. S., Molyneux, M., Murphy, G., Reid, S. J., Rummukainen, M., and Zerefos, C.: Chemical ozone loss in the arctic winter 1994/95 as determined by the match technique, J. Atmos. Chem., 32, 35-59, 1999.

Salsa, S.: Partial Differential Equations in Action: From Modelling to Theory, Springer, 2008.
Shutts, G., Kitchen, M., and Hoare, P.: A large amplitude gravity wave in the lower stratosphere detected by radiosonde, Q. J. Roy. Meteorol. Soc., 114, 579-594, doi:10.1002/qj.49711448103, 1988.

Son, K., Choi, J., Jeon, W., and Choi, H.: Effect of free-stream turbulence on the flow over a sphere, Phys. Fluids, 22, 045101, doi:10.1063/1.3371804, 2010.

Vargaftik, N., Flippov, Lev, P., and Tarzimanov, Amin, A.: Handbook of Thermal Conductivity of Liquids and Gases, CRC Press, ISBN: 0-8493-9345-0, 1994.

Veldhuis, C., Biesheuvel, A., and Lohse, D.: Freely rising light solid spheres, Int. J. Multiphas. Flow, 35, 312-322, doi:10.1016/j.ijmultiphaseflow.2009.01.005, 2009.

Vennard, J. K. and Street, R. L.: Elementary Fluid Mechanics, Wiley, 5th Edn., 1976.

Wang, J., Bian, Jianchun, Brown, William O., Cole, Harold, Grubišić, Vanda, Young, Kate: Vertical air motion from T-REX radiosonde and dropsonde data, J. Atmos. Ocean. Tech., 26, 928 942, 2009.

Yajima, N., Imamura, T., Izutsu, N., and Abe, T.: Scientific Ballooning, Springer, Berlin; ISBN: 978-0-387-09725-1, 2009.

Zink, F. and Vincent, R.: Wavelet analysis of stratospheric gravity wave packets over Macquarie Island - 2. Intermittency and mean-flow accelerations, J. Geophys. Res., 106, 10289-10297, 2001. 